\title{
The Stability of SI Epidemic Model in Complex Networks with Stochastic Perturbation
}

\author{
Jinqing Zhao, Maoxing Liu, Wanwan Wang, and Panzu Yang \\ Department of Mathematics, North University of China, Taiyuan, Shanxi 030051, China \\ Correspondence should be addressed to Maoxing Liu; liumaoxing@126.com
}

Received 29 November 2013; Accepted 12 January 2014; Published 13 March 2014

Academic Editor: Kaifa Wang

Copyright (C) 2014 Jinqing Zhao et al. This is an open access article distributed under the Creative Commons Attribution License, which permits unrestricted use, distribution, and reproduction in any medium, provided the original work is properly cited.

We investigate a stochastic SI epidemic model in the complex networks. We show that this model has a unique global positive solution. Then we consider the asymptotic behavior of the model around the disease-free equilibrium and show that the solution will oscillate around the disease-free equilibrium of deterministic system when $R_{0} \leq 1$. Furthermore, we derive that the disease will be persistent when $R_{0}>1$. Finally, a series of numerical simulations are presented to illustrate our mathematical findings. A new result is given such that, when $R_{0} \leq 1$, with the increase of noise intensity the solution of stochastic system converging to the disease-free equilibrium is faster than that of the deterministic system.

\section{Introduction}

Epidemiology is the science to study the distribution of disease and influencing factors, so as to explore the etiology, clarify the popular rule of the disease, and formulate the countermeasures and measures for preventing, controlling, and eliminating the disease. Many mathematical models of diseases spreading help us to understand the propagation of diseases $[1,2]$. The transmission of diseases can be influenced by many factors, such as the age and social structure of the population, the contact network among individuals, and the metapopulation characteristics. So it is difficult to establish an accurate epidemic model which is completely consistent with the real world. In recent years, a lot of compartmental epidemic models have been studied by many researchers [35], and complex networks also have been used to study the spread of diseases [6-17].

In this paper we consider an SI model with the birth and death in complex networks. As mentioned in the paper $[6,13]$, the birth and death do not affect the degree of nodes. Suppose $S_{k}(t), I_{k}(t)$ are the number of the healthy and infected nodes with the degree $k$ at time $t$; the mean-field equations can be written as

$$
\frac{d S_{k}}{d t}=b_{k}-\lambda k S_{k} \theta-d S_{k}
$$

$$
\frac{d I_{k}}{d t}=\lambda k S_{k} \theta-(d+\epsilon) I_{k},
$$

where $\theta=(1 /\langle k\rangle) \sum_{k=1}^{n} \lambda k P(k)$. For system (1), it can be written as the following form:

$$
\begin{aligned}
& d S_{k}(t)=\left(b_{k}-\frac{1}{\langle k\rangle} \sum_{j=1}^{n} \lambda k j P(j) S_{k}(t) I_{j}(t)-d S_{k}(t)\right) d t, \\
& d I_{k}(t)=\left(\frac{1}{\langle k\rangle} \sum_{j=1}^{n} \lambda k j P(j) S_{k}(t) I_{j}(t)-(d+\epsilon) I_{k}(t)\right) d t .
\end{aligned}
$$

We denote $\beta_{k j}=(1 /\langle k\rangle) \lambda k j P(j)$, so we obtain

$$
\begin{aligned}
& d S_{k}(t)=\left(b_{k}-\sum_{j=1}^{n} \beta_{k j} S_{k}(t) I_{j}(t)-d S_{k}(t)\right) d t, \\
& d I_{k}(t)=\left(\sum_{j=1}^{n} \beta_{k j} S_{k}(t) I_{j}(t)-(d+\epsilon) I_{k}(t)\right) d t .
\end{aligned}
$$

It always has the disease-free equilibrium $E_{0}=\left(S_{1}^{0}, 0, \ldots\right.$, $\left.S_{n}^{0}, 0\right)$, where $S_{k}^{0}=b_{k} / d, k=1,2, \ldots, n$. If $A=\left(\beta_{k j}\right)_{n \times n}$ is irreducible and $R_{0} \leq 1$, then $E_{0}$ is globally stable in $D$, while 
if $R_{0}>1, E_{0}$ is unstable and there is an endemic equilibrium $E^{*}=\left(S_{1}^{*}, I_{1}^{*}, \ldots, S_{n}^{*}, I_{n}^{*}\right)$ belonging to $D$ which is globally asymptotically stable in $D$; here

$$
\begin{aligned}
& D=\left\{\left(S_{1}, I_{1}, \ldots, S_{n}, I_{n}\right) \in \mathbb{R}_{+}^{2 n}: S_{k}, I_{k} \leq \frac{b_{k}}{d}, S_{k}+I_{k} \leq \frac{b_{k}}{d},\right. \\
& k=1,2, \ldots, n\}, \\
& M_{0}=M\left(S_{1}^{0}, \ldots, S_{n}^{0}\right)=\left(\frac{\beta_{k j} S_{k}^{0}}{d+\epsilon}\right)_{n \times n}, \quad R_{0}=\rho\left(M_{0}\right),
\end{aligned}
$$

and $\rho\left(M_{0}\right)$ denotes the spectral radius of $M_{0}$.

The deterministic models have some limitations in describing the spread of disease. The accident in the process of disease transmission can not be reflected by the deterministic models. This is because of the fact that the deterministic models ignore the effect of the environmental noise. In an ecosystem, the environmental noise is inevitably in the real world; thus stochastic models are more realistic. In the research of stochastic epidemic models, many researchers make a lot of contributions [17-26].

In this paper, we consider the following stochastic system:

$$
\begin{aligned}
d S_{k}(t)= & \left(b_{k}-\sum_{j=1}^{n} \beta_{k j} S_{k}(t) I_{j}(t)-d S_{k}(t)\right) d t \\
& +\sigma_{k 1} S_{k}(t) d B_{k 1}(t), \\
d I_{k}(t)= & \left(\sum_{j=1}^{n} \beta_{k j} S_{k}(t) I_{j}(t)-(d+\epsilon) I_{k}(t)\right) d t \\
& +\sigma_{k 2} I_{k}(t) d B_{k 2}(t),
\end{aligned}
$$

where $B_{k i}(t), k=1,2, \ldots, n, i=1,2$, are independent standard Brownian motions with $B_{k i}(0)=0$, and $\sigma_{k i}^{2} \geq 0$, $k=1,2, \ldots, n, i=1,2$, represent the intensities of $B_{k i}(t)$.

The remaining parts of this paper are as follows. In the next section we show the existence and uniqueness of a global positive solution of model (5). In Section 3, we analyze the asymptotic behavior around the disease-free equilibrium. In Section 4, we study the dynamic of system (5) around the endemic of the deterministic model. In Section 5, numerical simulations and conclusions are carried out.

\section{Global Positive Solution}

When we study a dynamical behavior, a global solution is important for the system. In this section we show that the solution of system (5) is global and nonnegative. As we know, for a stochastic differential equation, the coefficients of the equation are generally required to satisfy the linear growth condition and the local Lipschitz condition. It is a sufficient condition for a stochastic differential equation has a unique global (i.e., no explosion in a finite time) solution for any given initial value $[27,28]$. Although the coefficients of system
(5) satisfy locally Lipschitz continuous, they are not satisfied with the linear growth condition, so the solution of system (5) may explode at a finite time. In this section, Lyapunov analysis method (mentioned in [29]) is used to show that the solution of system (5) is positive and global.

Theorem 1. For any given initial value $\left(S_{1}(0), I_{1}(0), \ldots\right.$, $\left.S_{n}(0), I_{n}(0)\right) \in \mathbb{R}_{+}^{2 n}$, there is a unique positive solution $\left(S_{1}(t)\right.$, $\left.I_{1}(t), \ldots, S_{n}(t), I_{n}(t)\right)$ of model (5) on $t \geq 0$ and the solution will remain in $\mathbb{R}_{+}^{2 n}$ with probability 1 , namely, $\left(S_{1}(t)\right.$, $\left.I_{1}(t), \ldots, S_{n}(t), I_{n}(t)\right) \in \mathbb{R}_{+}^{2 n}$ for all $t \geq 0$ a.s.

Proof. Due to the fact that the coefficients of the system (5) are locally Lipschitz continuous, for any given initial value $\left(S_{1}(0), I_{1}(0), \ldots, S_{n}(0), I_{n}(0)\right) \in \mathbb{R}_{+}^{2 n}$, it has a unique local solution $\left(S_{1}(t), I_{1}(t), \ldots, S_{n}(t), I_{n}(t)\right)$ on $t \in\left[0, \tau_{e}\right)$, where $\tau_{e}$ is the explosion time [30]. If we show that $\tau_{e}=\infty$ a.s., it suggests that this solution is global. Let $l_{0}>0$ be sufficiently large so that $S_{k}(0), I_{k}(0)(k=1,2, \ldots, n)$ all lie within the interval $\left[1 / l_{0}, l_{0}\right]$. For each integer $l \geq l_{0}$, defining the stopping time

$$
\begin{gathered}
\tau_{l}=\inf \left\{t \in\left[0, \tau_{e}\right): \min \left\{S_{k}(t), I_{k}(t), k=1, \ldots, n\right\} \leq \frac{1}{l}\right. \\
\text { or } \left.\max \left\{S_{k}(t), I_{k}(t), k=1, \ldots, n\right\} \geq l\right\}
\end{gathered}
$$

we set $\inf \emptyset=\infty$ (as usual $\emptyset$ denotes the empty set). Obviously, $\tau_{l}$ is increasing as $l \rightarrow \infty$. Set $\tau_{\infty}=\lim _{l \rightarrow \infty} \tau_{l}$; therefore $\tau_{\infty} \leq \tau_{e}$ a.s. If $\tau_{\infty}=\infty$ a.s. is true, then $\tau_{e}=\infty$ a.s. and $\left(S_{1}(t), I_{1}(t), \ldots, S_{n}(t), I_{n}(t)\right) \in \mathbb{R}_{+}^{2 n}$ a.s. for $t \geq 0$. In other words, to complete the proof it is required to show that $\tau_{\infty}=\infty$ a.s. If this statement is false, then there is a pair of constants $T>0$ and $\varepsilon \in(0,1)$ such that $P\left\{\tau_{\infty} \leq T\right\}>\varepsilon$. Thus there is an integer $l_{1} \geq l_{0}$, such that

$$
P\left\{\tau_{l} \leq T\right\} \geq \varepsilon, \quad \forall l \geq l_{1}
$$

Define a $C^{2}$-function $V: \mathbb{R}_{+}^{2 n} \rightarrow \mathbb{R}_{+}$as follows:

$$
\begin{aligned}
V & \left(S_{1}, I_{1}, \ldots, S_{n}, I_{n}\right) \\
\quad & =\sum_{k=1}^{n}\left[\left(S_{k}-1-\ln S_{k}\right)+\left(I_{k}-1-\ln I_{k}\right)\right] .
\end{aligned}
$$

Applying Itô's formula, we obtain

$$
\begin{aligned}
d\left(S_{1}, I_{1}, \ldots, S_{n}, I_{n}\right) & \\
=\sum_{k=1}^{n}[ & \left(1-\frac{1}{S_{k}}\right) d S_{k}+\frac{1}{2} \frac{1}{S_{k}^{2}}\left(d S_{k}\right)^{2}+\left(1-\frac{1}{I_{k}}\right) d I_{k} \\
& \left.+\frac{1}{2} \frac{1}{I_{k}^{2}}\left(d I_{k}\right)^{2}\right]
\end{aligned}
$$




$$
\begin{aligned}
& =\sum_{k=1}^{n}\left[( 1 - \frac { 1 } { S _ { k } } ) \left[\left(b_{k}-\sum_{j=1}^{n} \beta_{k j} S_{k} I_{j}-d S_{k}\right) d t\right.\right. \\
& \left.+\sigma_{k 1} S_{k}(t) d B_{k 1}(t)\right] \\
& +\frac{1}{2} \sigma_{k 1}^{2} d t+\left(1-\frac{1}{I_{k}}\right) \\
& \times\left[\left(\sum_{j=1}^{n} \beta_{k j} S_{k} I_{j}-(d+\epsilon) I_{k}\right) d t+\sigma_{k 2} I_{k} d B_{k 2}(t)\right] \\
& \left.+\frac{1}{2} \sigma_{k 2}^{2} d t\right] \\
& =\sum_{k=1}^{n}\left[b_{k}-\sum_{j=1}^{n} \beta_{k j} S_{k} I_{j}-d S_{k}-\frac{b_{k}}{S_{k}}+\sum_{j=1}^{n} \beta_{k j} S_{k} I_{j}+d\right. \\
& +\frac{1}{2} \sigma_{k 1}^{2}+\sum_{j=1}^{n} \beta_{k j} S_{k} I_{j}-(d+\epsilon) I_{k}-\frac{\sum_{j=1}^{n} \beta_{k j} S_{k} I_{j}}{I_{k}} \\
& \left.+(d+\epsilon)+\frac{1}{2} \sigma_{k 2}^{2}\right] d t \\
& +\sum_{k=1}^{n}\left[\sigma_{k 1}\left(S_{k}-1\right) d B_{k 1}(t)+\sigma_{k 2} I_{k} d B_{k 2}(t)\right] \\
& \leq \sum_{k=1}^{n}\left(b_{k}+2 d+\epsilon+\sum_{j=1}^{n} \beta_{k j} I_{j}+\frac{1}{2}\left(\sigma_{k 1}^{2}+\sigma_{k 2}^{2}\right)\right) d t \\
& +\sum_{k=1}^{n}\left[\sigma_{k 1}\left(S_{k}-1\right) d B_{k 1}(t)+\sigma_{k 2}\left(I_{k}-1\right) d B_{k 2}(t)\right] \\
& =: K d t+\left[\sigma_{k 1}\left(S_{k}-1\right) d B_{k 1}(t)+\sigma_{k 2} I_{k} d B_{k 2}(t)\right] \text {. }
\end{aligned}
$$

We can now integrate both sides of (9) from 0 to $\tau_{l} \wedge T$ and then take the expectations

$$
\begin{aligned}
E & {\left[V\left(S_{1}\left(\tau_{l} \wedge T\right), I_{1}\left(\tau_{l} \wedge T\right), \ldots, S_{n}\left(\tau_{l} \wedge T\right), I_{n}\left(\tau_{l} \wedge T\right)\right)\right] } \\
& \leq V\left(S_{1}(0), I_{1}(0), \ldots, S_{n}(0), I_{n}(0)\right)+E\left[\int_{0}^{\tau_{l} \wedge T} K d t\right] \\
& \leq V\left(S_{1}(0), I_{1}(0), \ldots, S_{n}(0), I_{n}(0)\right)+K T .
\end{aligned}
$$

Let $\Omega_{l}=\left\{\tau_{l} \leq T\right\}$ for $l \geq l_{1}$ and, by (7), $P\left(\Omega_{l}\right) \geq$ $\varepsilon$. Note that, for every $\omega \in \Omega_{l}$, there is at least one of $S_{k}\left(\tau_{l}, \omega\right)$ and $I_{k}\left(\tau_{l}, \omega\right), k=1,2, \ldots, n$, that equals either $l$ or $1 / l$, and therefore $V\left(S_{1}\left(\tau_{l}, \omega\right), I_{1}\left(\tau_{l}, \omega\right), \ldots, S_{n}\left(\tau_{l}, \omega\right), I_{n}\left(\tau_{l}, \omega\right)\right)$ is not less than either

$$
l-1-\ln l \quad \text { or } \quad \frac{1}{l}-1-\ln \frac{1}{l}=\frac{1}{l}-1+\ln l .
$$

Hence,

$$
\begin{aligned}
& V\left(S_{1}\left(\tau_{l}, \omega\right), I_{1}\left(\tau_{l}, \omega\right), \ldots, S_{n}\left(\tau_{l}, \omega\right), I_{n}\left(\tau_{l}, \omega\right)\right) \\
& \quad \geq(l-1-\ln l) \wedge\left(\frac{1}{l}-1+\ln l\right) .
\end{aligned}
$$

It then follows from (7) and (10) that

$$
\begin{aligned}
& V\left(S_{1}(0), I_{1}(0), \ldots, S_{n}(0), I_{n}(0)\right)+K T \\
& \geq E\left[1_{\Omega_{l(\omega)}} V\left(S_{1}\left(\tau_{l}, \omega\right), I_{1}\left(\tau_{l}, \omega\right), \ldots, S_{n}\left(\tau_{l}, \omega\right), I_{n}\left(\tau_{l}, \omega\right)\right)\right] \\
& \geq \varepsilon\left[(l-1-\ln l) \wedge\left(\frac{1}{l}-1+\ln l\right)\right],
\end{aligned}
$$

where $1_{\Omega_{l(\omega)}}$ is the indicator function of $\Omega_{l}$. Letting $l \rightarrow \infty$, we have that

$$
\infty>V\left(S_{1}(0), I_{1}(0), \ldots, S_{n}(0), I_{n}(0)\right)+K T \geq \infty
$$

is a contradiction. So we must have $\tau_{\infty}=\infty$. Therefore, it implies $S_{k}(t), I_{k}(t), k=1,2, \ldots, n$, will not explode in a finite time with probability one.

\section{Asymptotic Behavior around the Disease-Free Equilibrium}

As mentioned in the Introduction, $E_{0}=\left(b_{1} / d, 0, \ldots, b_{n} / d, 0\right)$ is the disease-free equilibrium of system (3), and when $R_{0} \leq$ $1, E_{0}$ is globally stable, which means that the disease will be extinct in the limited time. In this section, we will study the asymptotic behavior around $E_{0}$ of system (5).

Lemma 2. If $A$ is nonnegative and irreducible, then the spectral radius $\rho(A)$ of $A$ is a simple eigenvalue, and $A$ has a positive eigenvector $\omega=\left(\omega_{1}, \omega_{2}, \ldots, \omega_{n}\right)$ corresponding to $\rho(A)$. Besides, if $0 \leq A \leq B$, then $\rho(A) \leq \rho(B)$. (This lemma can be found in [20].)

Theorem 3. Assume $A=\left(\beta_{k j}\right)_{n \times n}$ is irreducible. If $R_{0} \leq 1$ and the following condition is satisfied:

$$
\sigma_{k 1}^{2} \leq \frac{4}{3} d, \quad \sigma_{k 2}^{2} \leq 2(d+\epsilon)
$$

then for any given initial value $\left(S_{1}(0), I_{1}(0), \ldots, S_{n}(0), I_{n}(0)\right) \in$ $\mathbb{R}_{+}^{2 n}$, the solution of system (5) has the property

$$
\begin{aligned}
& \limsup _{t \rightarrow \infty} \frac{1}{t} E \int_{0}^{t}\left[\left(S_{k}(r)-\frac{b_{k}}{d}\right)^{2}+I_{k}^{2}(r)\right] d r \\
& \leq \frac{3}{2} \sum_{k=1}^{n} \frac{a_{k} \sigma_{k 1}^{2} b_{k}^{2}}{d^{2} K_{1}}
\end{aligned}
$$

where

$$
K_{1}=\min \left\{\frac{\omega_{k} \beta_{k k}}{(2 d+\epsilon)(d+\epsilon)}\left(d-\frac{3}{4} \sigma_{k 1}^{2}\right), d+\epsilon-\frac{1}{2} \sigma_{k 2}^{2}\right\} .
$$


Proof. First change the variables $s_{k}=S_{k}-b_{k} / d, i_{k}=I_{k}$; then $-b_{k} / d \leq s_{k} \leq 0, i_{k} \geq 0$ and system (5) can be written as

$$
\begin{gathered}
d s_{k}=\left(-\sum_{j=1}^{n} \beta_{k j}\left(s_{k}+\frac{b_{k}}{d}\right) i_{j}-d s_{k}\right) d t \\
+\sigma_{k 1}\left(s_{k}+\frac{b_{k}}{d}\right) d B_{k 1}(t), \\
d i_{k}=\left(\sum_{j=1}^{n} \beta_{k j}\left(s_{k}+\frac{b_{k}}{d}\right) i_{j}-(d+\epsilon) i_{k}\right) d t+\sigma_{k 2} i_{k} d B_{k 2}(t) .
\end{gathered}
$$

Let $S^{0}=\left(S_{1}^{0}, S_{2}^{0}, \ldots, S_{n}^{0}\right)$, where $S_{k}^{0}=b_{k} / d, k=1,2, \ldots, n$. Define

$$
M(s)=\left[\begin{array}{cccc}
\frac{\beta_{11} S_{1}^{0}}{d+\epsilon} & \frac{\beta_{12}\left(S_{1}^{0}+s_{1}\right)}{d+\epsilon} & \cdots & \frac{\beta_{1 n}\left(S_{1}^{0}+s_{1}\right)}{d+\epsilon} \\
\frac{\beta_{21}\left(S_{2}^{0}+s_{2}\right)}{d+\epsilon} & \frac{\beta_{22} S_{2}^{0}}{d+\epsilon} & \cdots & \frac{\beta_{2 n}\left(S_{2}^{0}+s_{2}\right)}{d+\epsilon} \\
\vdots & \vdots & \ddots & \vdots \\
\frac{\beta_{n 1}\left(S_{n}^{0}+s_{n}\right)}{d+\epsilon} & \frac{\beta_{n 2}\left(S_{n}^{0}+s_{n}\right)}{d+\epsilon} & \cdots & \frac{\beta_{n n} S_{n}^{0}}{d+\epsilon}
\end{array}\right] ;
$$

then it is nonnegative and irreducible. By Lemma 2, there is a positive eigenvector $\omega=\left(\omega_{1}, \omega_{2}, \ldots, \omega_{n}\right)$ of $M(s)$ corresponding to $\rho(M(s))$, such that

$$
\left(\omega_{1}, \omega_{2}, \ldots, \omega_{n}\right) M(s)=\left(\omega_{1}, \omega_{2}, \ldots, \omega_{n}\right) \rho(M(s)) .
$$

Define a $C^{2}$-function $V: \mathbb{R}_{+}^{2 n} \rightarrow \overline{\mathbb{R}}_{+}$by

$$
V\left(s_{1}, i_{1}, \ldots, s_{n}, i_{n}\right)=\frac{1}{2} \sum_{k=1}^{n} a_{k}\left(s_{k}+i_{k}\right)^{2}+\sum_{k=1}^{n} \frac{\omega_{k}}{d+\epsilon} i_{k}
$$

where $a_{k}, k=1,2, \ldots, n$, are positive constants. Then the function $V$ is positive definite, and

$$
\begin{aligned}
& d V=L V d t+\sum_{k=1}^{n} a_{k}\left(s_{k}+i_{k}\right) \\
& \quad \times\left(\sigma_{k 1}\left(s_{k}+\frac{b_{k}}{d}\right) d B_{k 1}(t)+\sigma_{k 2} i_{k} d B_{k 2}(t)\right) \\
& +\sum_{k=1}^{n} \frac{\omega_{k}}{d+\epsilon} \sigma_{k 2} i_{k} d B_{k 2}(t),
\end{aligned}
$$

where

$$
\begin{aligned}
L V= & \sum_{k=1}^{n} a_{k}\left(s_{k}+i_{k}\right)\left[-d s_{k}-(d+\epsilon) i_{k}\right] \\
& +\frac{1}{2} \sum_{k=1}^{n} a_{k}\left[\sigma_{k 1}^{2}\left(s_{k}+\frac{b_{k}}{d}\right)^{2}+\sigma_{k 2}^{2} i_{k}^{2}\right] \\
& +\sum_{k=1}^{n} \frac{\omega_{k}}{d+\epsilon}\left[\sum_{j=1}^{n} \beta_{k j}\left(s_{k}+\frac{b_{k}}{d}\right) i_{j}-(d+\epsilon) i_{k}\right] \\
= & -\sum_{k=1}^{n} a_{k}\left[\left(d-\frac{1}{2} \sigma_{k 1}^{2}\right) s_{k}^{2}+\left(d+\epsilon-\frac{1}{2} \sigma_{k 2}^{2}\right) i_{k}^{2}\right] \\
& +\sum_{k=1}^{n} \sum_{j \neq k} \frac{\omega_{k}}{d+\epsilon} \beta_{k j} s_{k} i_{k} \\
& -\sum_{k=1}^{n}\left[a_{k}(2 d+\epsilon)-\frac{\omega_{k}}{d+\epsilon} \beta_{k k}\right] s_{k} i_{k} \\
& +\sum_{k=1}^{n} \sum_{j=1}^{n} \frac{\omega_{k}}{d+\epsilon} \beta_{k j} \frac{b_{k}}{d} i_{k}-\sum_{k=1}^{n} \omega_{k} i_{k} \\
& +\frac{1}{2} \sum_{k=1}^{n} a_{k} \sigma_{k 1}^{2}\left(2 s_{k} \frac{b_{k}}{d}+\frac{b_{k}^{2}}{d^{2}}\right) .
\end{aligned}
$$

Choose $a_{k}=\omega_{k} \beta_{k k} /(2 d+\epsilon)(d+\epsilon), k=1,2, \ldots, n$; then $a_{k}(2 d+\epsilon)-\left(\omega_{k} /(d+\epsilon)\right) \beta_{k k}=0$. And we note that

$$
\begin{aligned}
\sum_{k=1}^{n} \sum_{j \neq k} \frac{\omega_{k}}{d+\epsilon} \beta_{k j} s_{k} i_{k}+\sum_{k=1}^{n} \sum_{j=1}^{n} \frac{\omega_{k}}{d+\epsilon} \beta_{k j} \frac{b_{k}}{d} i_{k} & \left(\omega_{1}, \omega_{2}, \ldots, \omega_{n}\right) \\
& \times\left[\begin{array}{cccc}
\frac{\beta_{11} S_{1}^{0}}{d+\epsilon} & \frac{\beta_{12}\left(S_{1}^{0}+s_{1}\right)}{d+\epsilon} & \cdots & \frac{\beta_{1 n}\left(S_{1}^{0}+s_{1}\right)}{d+\epsilon} \\
\frac{\beta_{21}\left(S_{2}^{0}+s_{2}\right)}{d+\epsilon} & \frac{\beta_{22} S_{2}^{0}}{d+\epsilon} & \ldots & \frac{\beta_{2 n}\left(S_{2}^{0}+s_{2}\right)}{d+\epsilon} \\
\vdots & \vdots & \ddots & \vdots \\
\frac{\beta_{n 1}\left(S_{n}^{0}+s_{n}\right)}{d+\epsilon} & \frac{\beta_{n 2}\left(S_{n}^{0}+s_{n}\right)}{d+\epsilon} & \ldots & \frac{\beta_{n n} S_{n}^{0}}{d+\epsilon}
\end{array}\right] \\
& \times\left[\begin{array}{c}
i_{1} \\
i_{2} \\
\vdots \\
i_{n}
\end{array}\right] \\
= & \omega M(s) i .
\end{aligned}
$$


Then

$$
\begin{aligned}
L V= & -\sum_{k=1}^{n} a_{k}\left[\left(d-\frac{1}{2} \sigma_{k 1}^{2}\right) s_{k}^{2}+\left(d+\epsilon-\frac{1}{2} \sigma_{k 2}^{2}\right) i_{k}^{2}\right] \\
& -\omega i+\omega M(s) i+\frac{1}{2} \sum_{k=1}^{n} a_{k} \sigma_{k 1}^{2}\left(2 s_{k} \frac{b_{k}}{d}+\frac{b_{k}^{2}}{d^{2}}\right) \\
\leq & -\sum_{k=1}^{n} a_{k}\left[\left(d-\frac{3}{4} \sigma_{k 1}^{2}\right) s_{k}^{2}+\left(d+\epsilon-\frac{1}{2} \sigma_{k 2}^{2}\right) i_{k}^{2}\right] \\
& +\omega(M(s)-1) i+\frac{3}{2} \sum_{k=1}^{n} a_{k} \sigma_{k 1}^{2} \frac{b_{k}^{2}}{d^{2}} \\
= & -\sum_{k=1}^{n} a_{k}\left[\left(d-\frac{3}{4} \sigma_{k 1}^{2}\right) s_{k}^{2}+\left(d+\epsilon-\frac{1}{2} \sigma_{k 2}^{2}\right) i_{k}^{2}\right] \\
& +\omega(\rho(M(s))-1) i+\frac{3}{2} \sum_{k=1}^{n} a_{k} \sigma_{k 1}^{2} \frac{b_{k}^{2}}{d^{2}},
\end{aligned}
$$

where the last equality is derived from (20). Since $-b_{k} / d \leq$ $s_{k} \leq 0$, then $0 \leq M(s) \leq M\left(S^{0}\right)=\left(S_{k}^{0} \beta_{k j} /(d+\epsilon)\right)_{n \times n}=$ $M_{0}$, and so $\rho(M(s)) \leq \rho\left(M_{0}\right)$ according to Lemma 2. Besides, $R_{0} \leq 1$, and then $\rho(M(s)) \leq 1$. Therefore

$$
\begin{aligned}
d V \leq & -\sum_{k=1}^{n} a_{k}\left[\left(d-\frac{3}{4} \sigma_{k 1}^{2}\right) s_{k}^{2}+\left(d+\epsilon-\frac{1}{2} \sigma_{k 2}^{2}\right) i_{k}^{2}\right] \\
& \left.+\frac{3}{2} \sum_{k=1}^{n} a_{k} \sigma_{k 1}^{2} \frac{b_{k}^{2}}{d^{2}}\right] d t \\
& +\sum_{k=1}^{n} a_{k}\left(s_{k}+i_{k}\right) \sigma_{k 1}\left(s_{k}+\frac{b_{k}}{d}\right) d B_{k 1}(t) \\
& +\sum_{k=1}^{n}\left(a_{k}\left(s_{k}+i_{k}\right) \sigma_{k 2}+\frac{\omega_{k}}{d+\epsilon} \sigma_{k 2}\right) i_{k} d B_{k 2}(t) .
\end{aligned}
$$

Integrating both sides of (26) from 0 to $t$, and taking expectation, yields

$$
\begin{gathered}
0 \leq E\left[V\left(s_{1}(t), i_{1}(t), \ldots, s_{n}(t), i_{n}(t)\right)\right] \\
\leq E\left[V\left(s_{1}(0), i_{1}(0), \ldots, s_{n}(0), i_{n}(0)\right)\right] \\
+E \int_{0}^{t}\left[-\sum_{k=1}^{n} a_{k}\left[\left(d-\frac{3}{4} \sigma_{k 1}^{2}\right) s_{k}^{2}(r)\right.\right. \\
\left.+\left(d+\epsilon-\frac{1}{2} \sigma_{k 2}^{2}\right) i_{k}^{2}(r)\right] \\
\left.+\frac{3}{2} \sum_{k=1}^{n} a_{k} \sigma_{k 1}^{2} \frac{b_{k}^{2}}{d^{2}}\right] d r,
\end{gathered}
$$

which implies

$$
\begin{gathered}
E \int_{0}^{t}\left[\sum_{k=1}^{n} a_{k}\left[\left(d-\frac{3}{4} \sigma_{k 1}^{2}\right) s_{k}^{2}(r)+\left(d+\epsilon-\frac{1}{2} \sigma_{k 2}^{2}\right) i_{k}^{2}(r)\right]\right] d r \\
\leq E\left[V\left(s_{1}(0), i_{1}(0), \ldots, s_{n}(0), i_{n}(0)\right)\right]+\frac{3}{2} \sum_{k=1}^{n} a_{k} \sigma_{k 1}^{2} \frac{b_{k}^{2}}{d^{2}} t .
\end{gathered}
$$

Therefore

$$
\begin{gathered}
\limsup _{t \rightarrow \infty} \frac{1}{t} E \int_{0}^{t}\left[\sum _ { k = 1 } ^ { n } a _ { k } \left[\left(d-\frac{3}{4} \sigma_{k 1}^{2}\right) s_{k}^{2}(r)\right.\right. \\
\left.\left.+\left(d+\epsilon-\frac{1}{2} \sigma_{k 2}^{2}\right) i_{k}^{2}(r)\right]\right] d r \\
\leq \frac{3}{2} \sum_{k=1}^{n} a_{k} \sigma_{k 1}^{2} \frac{b_{k}^{2}}{d^{2}}
\end{gathered}
$$

that is,

$$
\begin{gathered}
\limsup _{t \rightarrow \infty} \frac{1}{t} E \int_{0}^{t}\left[\sum _ { k = 1 } ^ { n } a _ { k } \left[\left(d-\frac{3}{4} \sigma_{k 1}^{2}\right)\left(S_{k}(r)-\frac{b_{k}}{d}\right)^{2}\right.\right. \\
\left.\left.+\left(d+\epsilon-\frac{1}{2} \sigma_{k 2}^{2}\right) I_{k}^{2}(r)\right]\right] d r \\
\leq \frac{3}{2} \sum_{k=1}^{n} a_{k} \sigma_{k 1}^{2} \frac{b_{k}^{2}}{d^{2}} .
\end{gathered}
$$

If we let

$$
K_{1}=\min \left\{a_{k}\left(d-\frac{3}{4} \sigma_{k 1}^{2}\right), d+\epsilon-\frac{1}{2} \sigma_{k 2}^{2}\right\},
$$

then

$$
\begin{aligned}
& \limsup _{t \rightarrow \infty} \frac{1}{t} E \int_{0}^{t}\left[\left(S_{k}(r)-\frac{b_{k}}{d}\right)^{2}+I_{k}^{2}(r)\right] d r \\
& \leq \frac{3}{2} \sum_{k=1}^{n} \frac{a_{k} \sigma_{k 1}^{2} b_{k}^{2}}{d^{2} K_{1}},
\end{aligned}
$$

as the theorem is proved.

Remark 4. From Theorem 3, we can get the conclusion that the solution of the stochastic system will oscillate around the disease-free equilibrium of the deterministic model; the values of $\sigma_{k 1}$ and $\sigma_{k 2}$ have bearing on the intensity of turbulence. If the stochastic perturbations become small, the solution of system (5) will be close to the disease-free equilibrium of system (3).

Besides, if $\sigma_{k 1}=0$, then $E_{0}$ is also the disease-free equilibrium of system (5). From the proof of Theorem 3, we can obtain

$$
L V \leq-\sum_{k=1}^{n} a_{k}\left[\left(d-\frac{3}{4} \sigma_{k 1}^{2}\right) s_{k}^{2}+\left(d+\epsilon-\frac{1}{2} \sigma_{k 2}^{2}\right) i_{k}^{2}\right] \leq 0 .
$$

Therefore, $E_{0}$ is globally asymptotically stable. 


\section{The Dynamic of System (5) around the Endemic of System (3)}

In the deterministic model, if $R_{0}>1$, there exists the endemic equilibrium $E^{*}$. But $E^{*}$ is not the endemic equilibrium of stochastic system (5), because there is no endemic equilibrium for the stochastic system (5). In fact, we still want to find the relation between the solution of stochastic system and $E^{*}$.

Given a weighted digraph $(\mathscr{G}, A)$ with $n$ vertices, where $A=\left(a_{k j}\right)_{n n}$ is the weight matrix, whose entry $a_{k j}$ equals the weight of $\operatorname{arc}(j, k)$ if it exists, and 0 otherwise, the Laplacian matrix of $A$ is defined as

$$
L_{A}=\left[\begin{array}{cccc}
\sum_{k \neq 1} a_{1 k} & -a_{12} & \cdots & -a_{1 n} \\
-a_{21} & \sum_{k \neq 2} a_{2 k} & \cdots & -a_{2 n} \\
\vdots & \vdots & \ddots & \vdots \\
-a_{n 1} & -a_{n 2} & \cdots & \sum_{k \neq n} a_{n k}
\end{array}\right]
$$

Let $c_{k}$ denote the cofactor of the $k$ th diagonal element of $L_{A}$, and we have the following results.

Theorem 5. Assume $A=\left(\beta_{k j}\right)_{n \times n}$ is irreducible and $R_{0}>$ 1. For any given initial value $\left(S_{1}(0), I_{1}(0), \ldots, S_{n}(0), I_{n}(0)\right) \in$ $\mathbb{R}_{+}^{2 n}$, the solution of system (5) has the property

$$
\begin{aligned}
\limsup _{t \rightarrow \infty} \frac{1}{t} \sum_{k=1}^{n} \int_{0}^{t}\left[p_{k} d\left(S_{k}-S_{k}^{*}\right)^{2}+m_{k}(d+\epsilon)\left(I_{k}-I_{k}^{*}\right)^{2}\right] d s \\
\leq \sum_{k=1}^{n}\left[\left(\frac{a \bar{c}_{k} b_{k}}{d}+\frac{\left(m_{k}+p_{k}\right) b_{k}^{2}}{d^{2}}\right) \sigma_{k 1}^{2}\right. \\
\left.+\left(\frac{(a+1) \bar{c}_{k} b_{k}}{d}+\frac{m_{k} b_{k}^{2}}{d^{2}}\right) \sigma_{k 2}^{2}\right], \quad \text { a.s., }
\end{aligned}
$$

where $E^{*}=\left(S_{1}^{*}, I_{1}^{*}, \ldots, S_{n}^{*}, I_{n}^{*}\right)$ is the endemic equilibrium of system (3) and $\bar{c}_{k}, k=1,2, \ldots, n$, denote the cofactor of the $k t h$ diagonal element of $L_{\bar{A}}\left(\bar{A}=\left(\bar{\beta}_{k j}\right)_{n \times n}=\left(\beta_{k j} S_{k}^{*} I_{j}^{*}\right)_{n \times n}\right)$, and $a$, $m_{k}, p_{k}, k=1,2, \ldots, n$, are positive constants defined as in the proof.

Proof. Since $E^{*}$ is the endemic equilibrium of system (3), we have

$$
\sum_{j=1}^{n} \beta_{k j} S_{k}^{*} I_{j}^{*}+d S_{k}^{*}=b_{k}, \quad \sum_{j=1}^{n} \beta_{k j} S_{k}^{*} I_{j}^{*}=(d+\epsilon) I_{k}^{*} .
$$

Define

$$
\begin{aligned}
V & \left(S_{1}, I_{1}, \ldots, S_{n}, I_{n}\right) \\
& =a \sum_{k=1}^{n} \bar{c}_{k}\left(S_{k}-S_{k}^{*}-S_{k}^{*} \ln \frac{S_{k}}{S_{k}^{*}}+I_{k}-I_{k}^{*}-I_{k}^{*} \ln \frac{I_{k}}{I_{k}^{*}}\right)
\end{aligned}
$$

$$
\begin{aligned}
& +\sum_{k=1}^{n} \bar{c}_{k}\left(I_{k}-I_{k}^{*}-I_{k}^{*} \ln \frac{I_{k}}{I_{k}^{*}}\right) \\
& +\frac{1}{2} \sum_{k=1}^{n} m_{k}\left(S_{k}-S_{k}^{*}+I_{k}-I_{k}^{*}\right)^{2}+\frac{1}{2} \sum_{k=1}^{n} p_{k}\left(S_{k}-S_{k}^{*}\right)^{2} \\
:= & a V_{1}+V_{2}+V_{3}+V_{4},
\end{aligned}
$$

where $a, m_{k}, p_{k}, k=1,2, \ldots, n$, are positive constants to be determined later. From the property (1) of Lemma A.2 (see [20]), we know $\bar{c}_{k}>0, k=1,2, \ldots, n$. Hence $V$ is positive definite. Let $L$ be the generating operator of system (5). Then we get

$$
\begin{aligned}
& L V_{1}=\sum_{k=1}^{n} \bar{c}_{k}\left(1-\frac{S_{k}^{*}}{S_{k}}\right)\left(b_{k}-\sum_{j=1}^{n} \beta_{k j} S_{k} I_{j}-d S_{k}\right) \\
& +\sum_{k=1}^{n} \frac{\bar{c}_{k} S_{k}^{*} \sigma_{k 1}^{2}}{2} \\
& +\sum_{k=1}^{n} \bar{c}_{k}\left(1-\frac{I_{k}^{*}}{I_{k}}\right)\left(\sum_{j=1}^{n} \beta_{k j} S_{k} I_{j}-(d+\epsilon) I_{k}\right) \\
& +\sum_{k=1}^{n} \frac{\bar{c}_{k} I_{k}^{*} \sigma_{k 2}^{2}}{2} \\
& =\sum_{k=1}^{n} \bar{c}_{k}\left[b_{k}-d S_{k}-b_{k} \frac{S_{k}^{*}}{S_{k}}+\sum_{j=1}^{n} \beta_{k j} S_{k}^{*} I_{j}+d S_{k}^{*}\right. \\
& -(d+\epsilon) I_{k}+(d+\epsilon) I_{k}^{*}-\sum_{j=1}^{n} \beta_{k j} \frac{S_{k} I_{j} I_{k}^{*}}{I_{k}} \\
& \left.+\frac{1}{2}\left(S_{k}^{*} \sigma_{k 1}^{2}+I_{k}^{*} \sigma_{k 2}^{2}\right)\right] \\
& =\sum_{k=1}^{n} \bar{c}_{k}\left[\sum_{j=1}^{n} \bar{\beta}_{k j}+d S_{k}^{*}-d S_{k}-\sum_{j=1}^{n} \bar{\beta}_{k j} \frac{S_{k}^{*}}{S_{k}}-d S_{k}^{*} \frac{S_{k}^{*}}{S_{k}}\right. \\
& +\sum_{j=1}^{n} \bar{\beta}_{k j} \frac{I_{j}}{I_{j}^{*}}+d S_{k}^{*}-\sum_{j=1}^{n} \bar{\beta}_{k j} \frac{I_{k}}{I_{k}^{*}}-\sum_{j=1}^{n} \bar{\beta}_{k j} \frac{S_{k} I_{j} I_{k}^{*}}{I_{k} S_{k}^{*} I_{j}^{*}} \\
& \left.+\sum_{j=1}^{n} \bar{\beta}_{k j}+\frac{1}{2}\left(S_{k}^{*} \sigma_{k 1}^{2}+I_{k}^{*} \sigma_{k 2}^{2}\right)\right] \\
& =\sum_{k=1}^{n} \bar{c}_{k}\left[-d S_{k}^{*}\left(\frac{S_{k}^{*}}{S_{k}}+\frac{S_{k}}{S_{k}^{*}}-2\right)+\sum_{j=1}^{n} \bar{\beta}_{k j}\left(\frac{I_{j}}{I_{j}^{*}}-\frac{I_{k}}{I_{k}^{*}}\right)\right. \\
& +\sum_{j=1}^{n} \bar{\beta}_{k j}\left(2-\frac{S_{k}^{*}}{S_{k}}-\frac{S_{k} I_{j} I_{k}^{*}}{I_{k} S_{k}^{*} I_{j}^{*}}\right) \\
& \left.+\frac{1}{2}\left(S_{k}^{*} \sigma_{k 1}^{2}+I_{k}^{*} \sigma_{k 2}^{2}\right)\right] \text {. }
\end{aligned}
$$



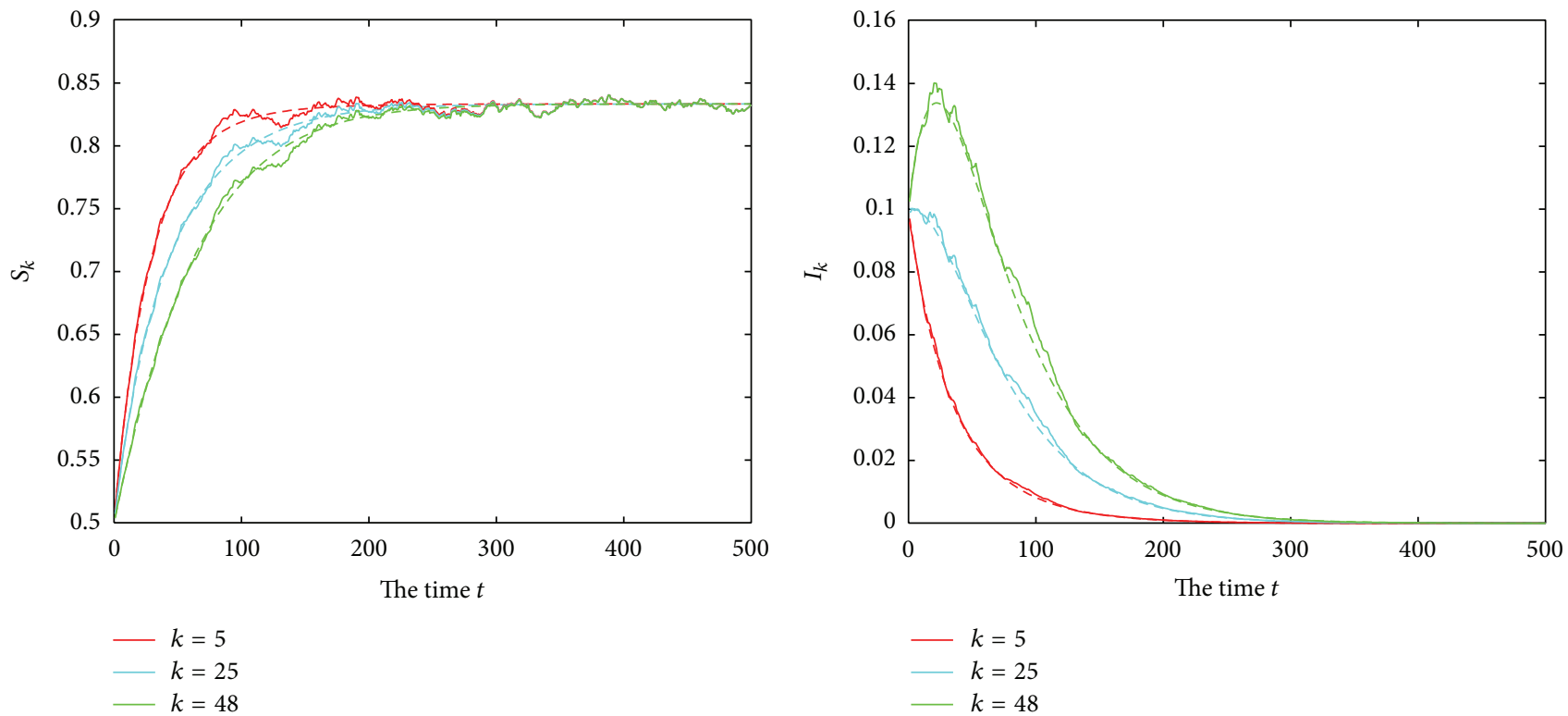

(a)
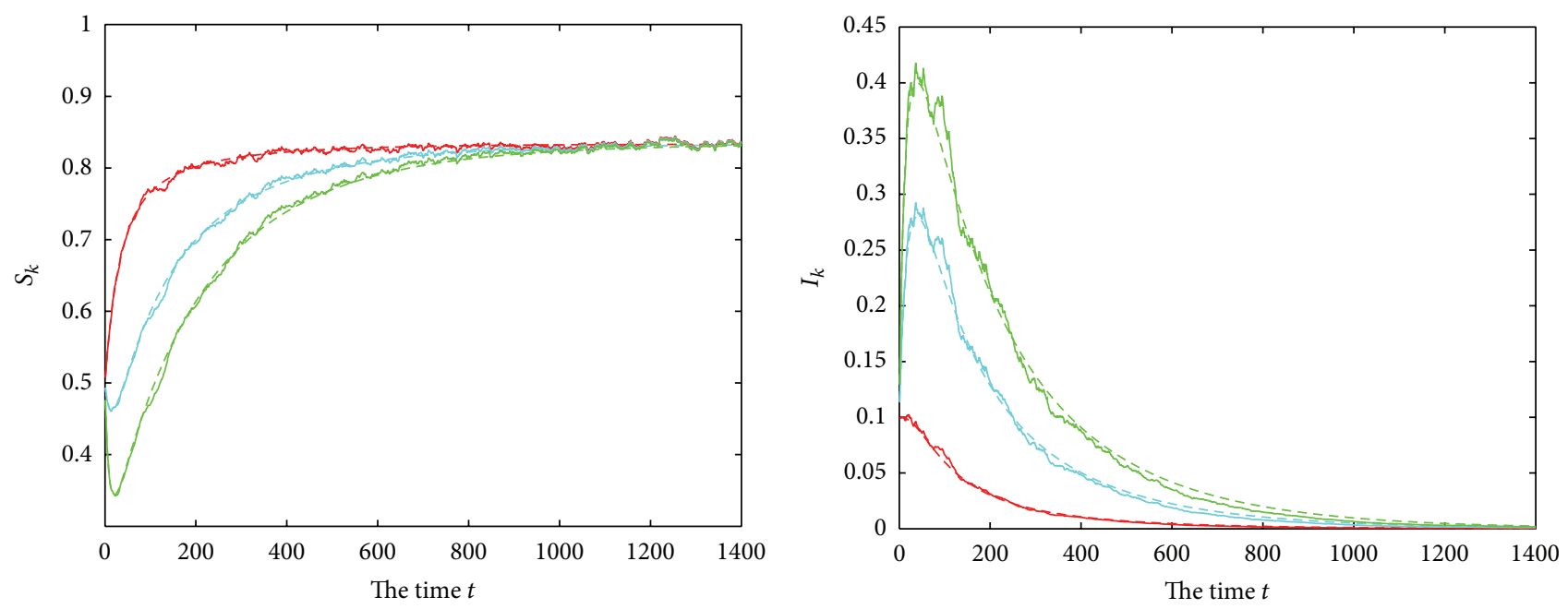

$$
\begin{aligned}
-k & =5 \\
-k & =25 \\
k & =48
\end{aligned}
$$

$$
\begin{aligned}
-k & =5 \\
-k & =25 \\
-k & =48
\end{aligned}
$$

(c)

(d)

FigURE 1: $\lambda=0.025, \sigma_{k 1}=0.005, \sigma_{k 2}=0.03, R_{0} \leq 1$. (a), (b) $P(k)=m^{k} \exp (-m) / k !, m=6, R_{0}=0.4704 \leq 1$; (c), (d) $P(k)=2 m^{2} k^{-3}, m=3$, $R_{0}=0.9071 \leq 1$.

$$
\begin{array}{rlr}
L V_{2}=\sum_{k=1}^{n} \bar{c}_{k}\left(1-\frac{I_{k}^{*}}{I_{k}}\right)\left(\sum_{j=1}^{n} \beta_{k j} S_{k} I_{j}-(d+\epsilon) I_{k}\right) & =\sum_{k=1}^{n} \bar{c}_{k}\left[\sum_{j=1}^{n} \beta_{k j} S_{k} I_{j}-\sum_{j=1}^{n} \bar{\beta}_{k j} \frac{I_{k}}{I_{k}^{*}}-\sum_{j=1}^{n} \bar{\beta}_{k j} \frac{S_{k} I_{j} I_{k}^{*}}{I_{k} S_{k}^{*} I_{j}^{*}}\right. \\
+\sum_{k=1}^{n} \frac{\bar{c}_{k} I_{k}^{*} \sigma_{k 2}^{2}}{2} & \left.+\sum_{j=1}^{n} \bar{\beta}_{k j}+\frac{I_{k}^{*} \sigma_{k 2}^{2}}{2}\right] . \\
=\sum_{k=1}^{n} \bar{c}_{k}\left[\sum_{j=1}^{n} \beta_{k j} S_{k} I_{j}-(d+\epsilon) I_{k}-\frac{I_{k}^{*}}{I_{k}} \sum_{j=1}^{n} \beta_{k j} S_{k} I_{j}\right. & L V_{3}=\sum_{k=1}^{n} m_{k}\left(S_{k}-S_{k}^{*}+I_{k}-I_{k}^{*}\right)\left(b_{k}-d S_{k}-(d+\epsilon) I_{k}\right) \\
\left.+(d+\epsilon) I_{k}^{*}+\frac{I_{k}^{*} \sigma_{k 2}^{2}}{2}\right] & +\sum_{k=1}^{n} m_{k} \frac{\sigma_{k 1}^{2} S_{k}^{2}+\sigma_{k 2}^{2} I_{k}^{2}}{2}
\end{array}
$$



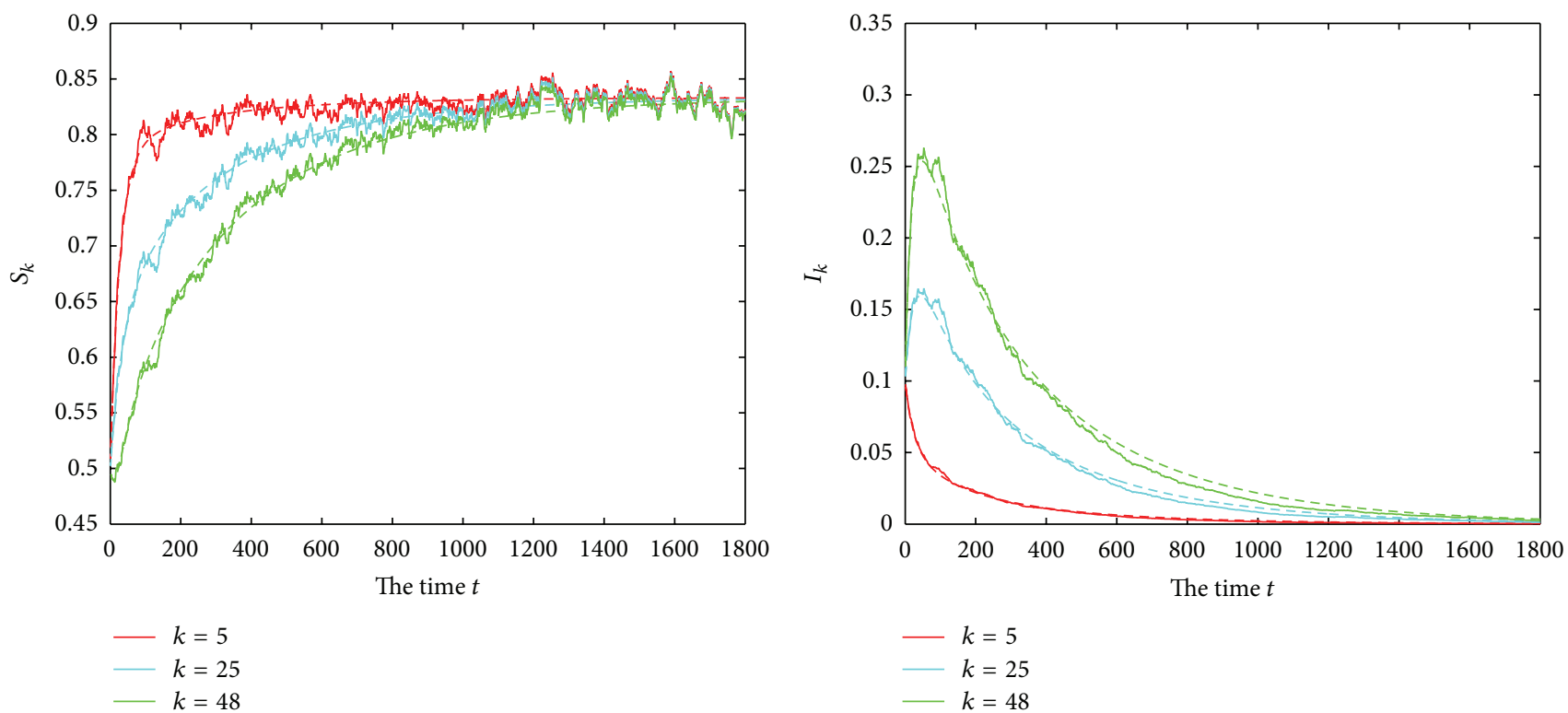

(a)

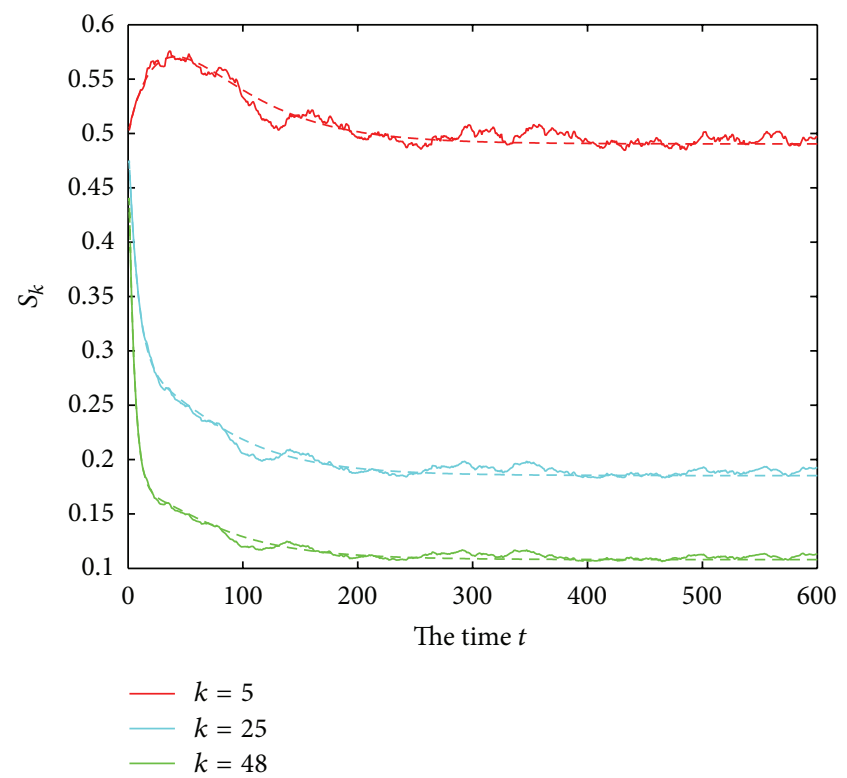

(c)

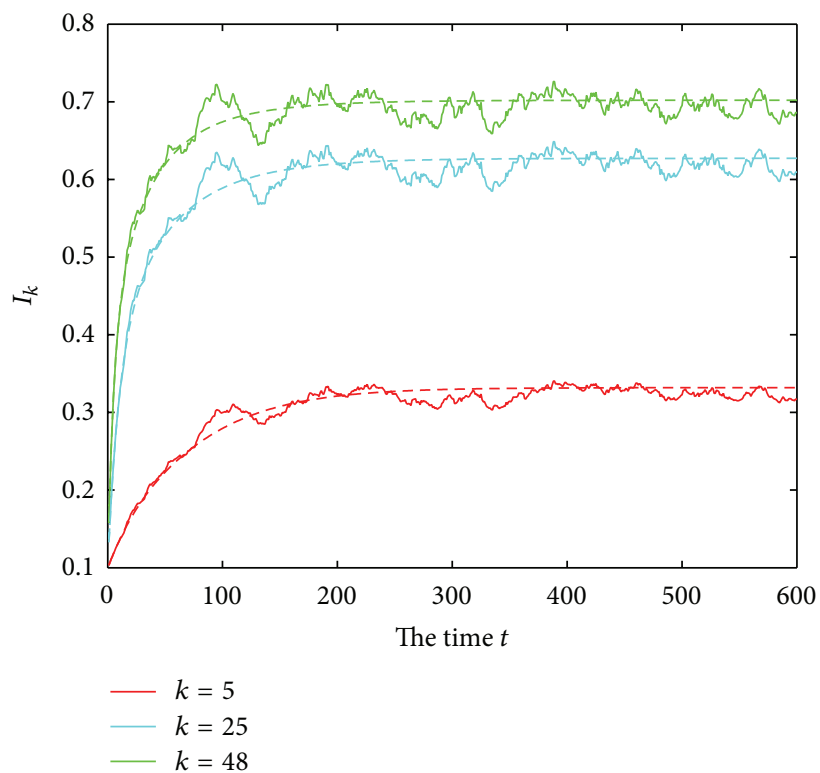

(d)

FIGURE 2: $\lambda=0.05, \sigma_{k 1}=0.01, \sigma_{k 2}=0.02$. (a), (b) $P(k)=m^{k} \exp (-m) / k !, m=6, R_{0}=0.9409 \leq 1$; (c), (d) $P(k)=2 m^{2} k^{-3}, m=3$, $R_{0}=1.8142 \geq 1$.

$$
\begin{gathered}
=\sum_{k=1}^{n} m_{k}\left(S_{k}-S_{k}^{*}+I_{k}-I_{k}^{*}\right) \\
\times\left(-d\left(S_{k}-S_{k}^{*}\right)-(d+\epsilon)\left(I_{k}-I_{k}^{*}\right)\right) \\
+\sum_{k=1}^{n} m_{k} \frac{\sigma_{k 1}^{2} S_{k}^{2}+\sigma_{k 2}^{2} I_{k}^{2}}{2} \\
=\sum_{k=1}^{n} m_{k}\left[-d\left(S_{k}-S_{k}^{*}\right)^{2}-(d+\epsilon)\left(I_{k}-I_{k}^{*}\right)^{2}\right. \\
-(2 d+\epsilon)\left(S_{k}-S_{k}^{*}\right)\left(I_{k}-I_{k}^{*}\right) \\
\left.+\frac{\sigma_{k 1}^{2} S_{k}^{2}+\sigma_{k 2}^{2} I_{k}^{2}}{2}\right]
\end{gathered}
$$

$$
\begin{gathered}
\leq \sum_{k=1}^{n} m_{k}\left[-\left(d-\frac{(2 d+\epsilon)^{2}}{2(d+\epsilon)}\right)\left(S_{k}-S_{k}^{*}\right)^{2}\right. \\
\left.-\frac{(d+\epsilon)}{2}\left(I_{k}-I_{k}^{*}\right)^{2}+\frac{\sigma_{k 1}^{2} S_{k}^{2}+\sigma_{k 2}^{2} I_{k}^{2}}{2}\right] \\
=\sum_{k=1}^{n} m_{k}\left[\frac{(d+\epsilon)^{2}+d^{2}}{2(d+\epsilon)}\left(S_{k}-S_{k}^{*}\right)^{2}-\frac{(d+\epsilon)}{2}\left(I_{k}-I_{k}^{*}\right)^{2}\right. \\
\left.+\frac{\sigma_{k 1}^{2} S_{k}^{2}+\sigma_{k 2}^{2} I_{k}^{2}}{2}\right] .
\end{gathered}
$$



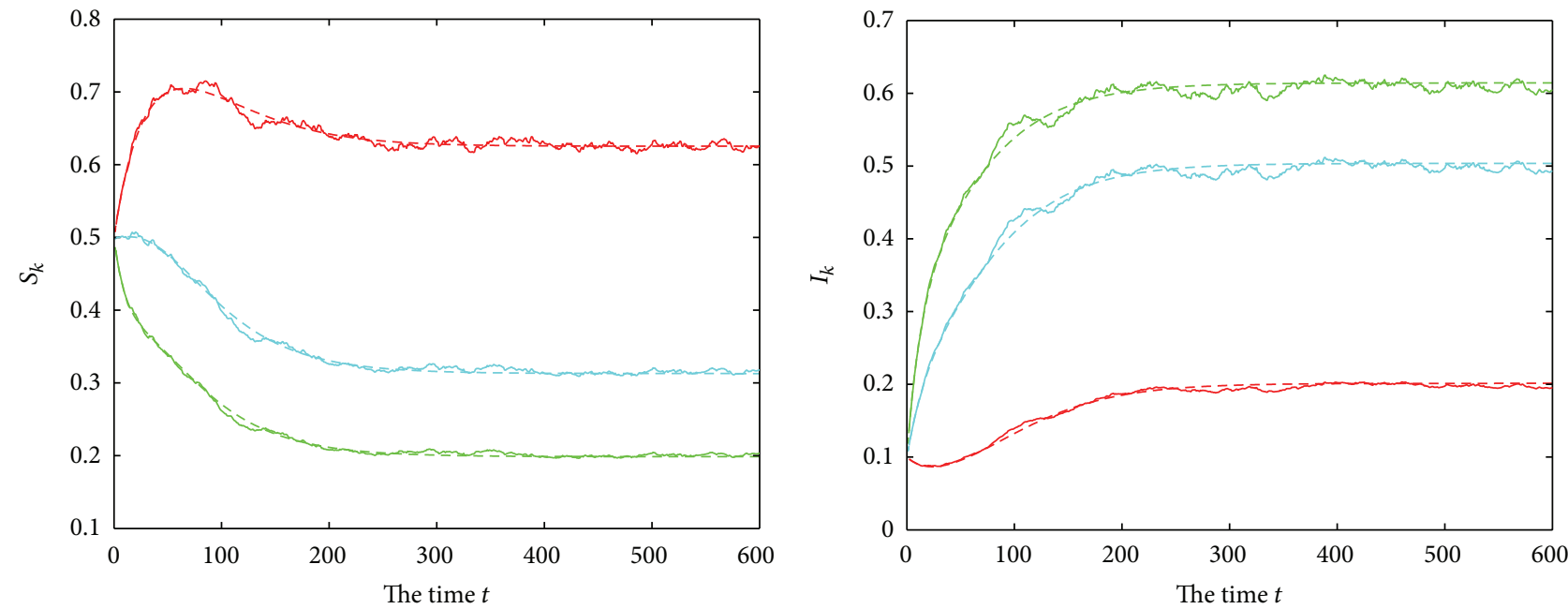

$\begin{aligned} k & =5 \\ -k & =25\end{aligned}$

$k=5$

- $k=25$

- $k=48$

(a)
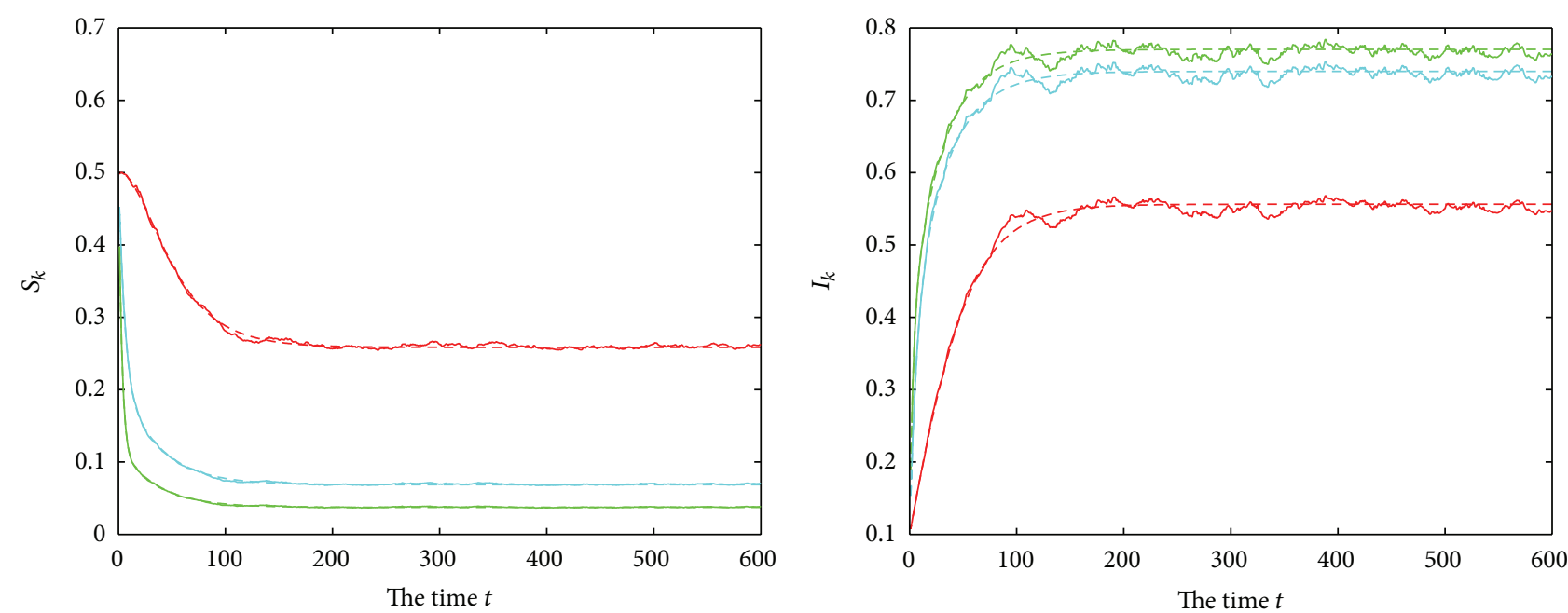

$\begin{aligned} k & =5 \\ -k & =25 \\ -k & =48\end{aligned}$

- $k=5$

$-k=25$

$-k=48$

(c)

(d)

FigURE 3: $\lambda=0.08, \sigma_{k 1}=0.01, \sigma_{k 2}=0.01, R_{0} \geq 1$. (a), (b) $P(k)=m^{k} \exp (-m) / k !, m=6, R_{0}=1.5054 \geq 1$; (c), (d) $P(k)=2 m^{2} k^{-3}, m=3$, $R_{0}=2.9027 \geq 1$.

$$
\begin{array}{rlr}
L V_{4}=\sum_{k=1}^{n} p_{k}\left(S_{k}-S_{k}^{*}\right)\left(b_{k}-\sum_{j=1}^{n} \beta_{k j} S_{k} I_{j}-d S_{k}\right) & +\sum_{k=1}^{n} p_{k} \frac{\sigma_{k 1}^{2} S_{k}^{2}}{2} \\
& +\sum_{k=1}^{n} p_{k} \frac{\sigma_{k 1}^{2} S_{k}^{2}}{2} & =-\sum_{k=1}^{n} p_{k}\left[\sum_{j=1}^{n} \beta_{k j} S_{k}^{*}\left(S_{k}-S_{k}^{*}\right)\left(I_{j}-I_{j}^{*}\right)\right. \\
=\sum_{k=1}^{n} p_{k}\left(S_{k}-S_{k}^{*}\right) & \left.+\sum_{j=1}^{n} \beta_{k j} I_{j}\left(S_{k}-S_{k}^{*}\right)^{2}+d\left(S_{k}-S_{k}^{*}\right)^{2}-\frac{\sigma_{k 1}^{2} S_{k}^{2}}{2}\right] . \\
\quad \times\left(\sum_{j=1}^{n} \beta_{k j}\left(S_{k}^{*} I_{j}^{*}-S_{k} I_{j}\right)-d\left(S_{k}-S_{k}^{*}\right)\right) &
\end{array}
$$



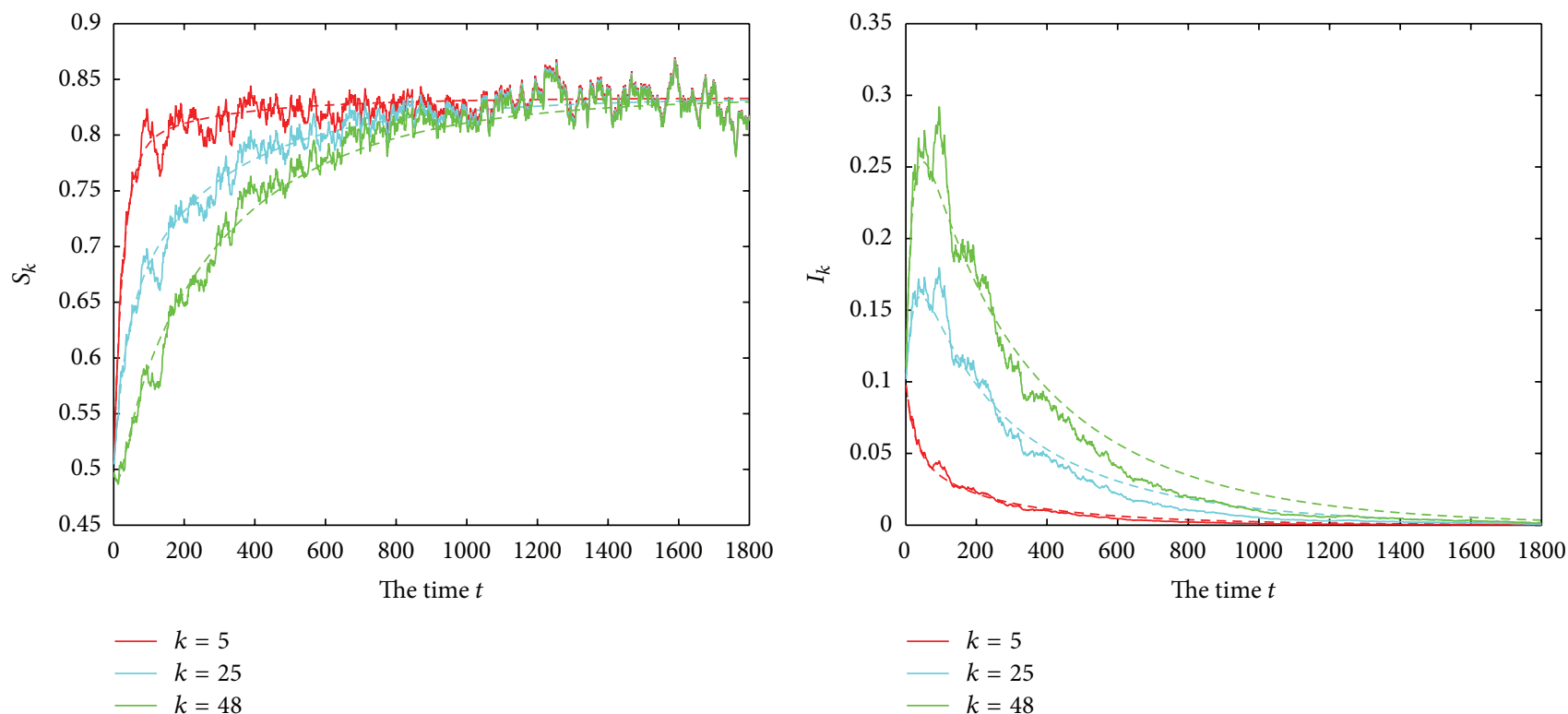

(a)

(b)
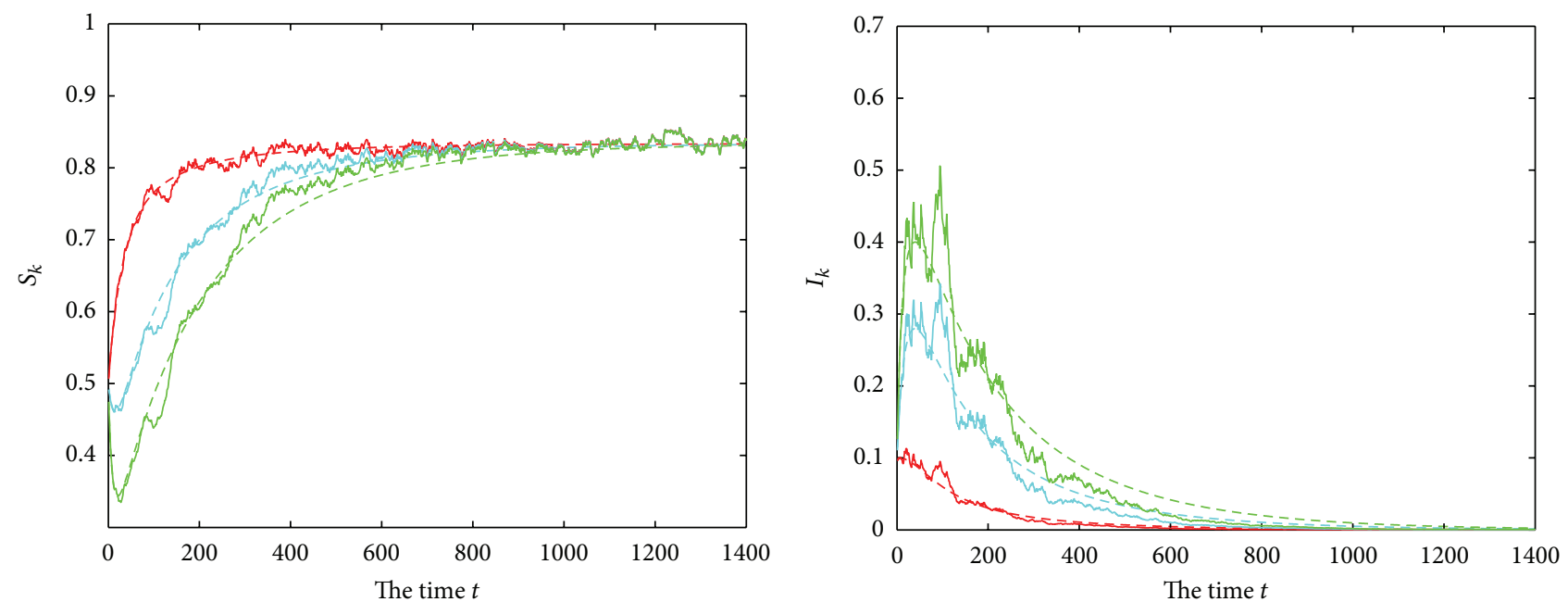

$$
\begin{aligned}
k & =5 \\
k & =25
\end{aligned}
$$$$
-k=5
$$$$
k=25
$$$$
-k=48
$$

(c)

Figure 4: (a), (b) $\lambda=0.05, \sigma_{k 1}=0.015, \sigma_{k 2}=0.05, P(k)=m^{k} \exp (-m) / k !, m=6, R_{0}=0.94 .9 \leq 1$. (c), (d) $\lambda=0.025, \sigma_{k 1}=0.01, \sigma_{k 2}=0.1$, $P(k)=2 m^{2} k^{-3}, m=3, R_{0}=0.9071 \leq 1$.

By property (2) of Lemma A.2 (see [20]), we know

$$
\begin{gathered}
\sum_{k=1}^{n} \bar{c}_{k}\left(\sum_{j=1}^{n} \bar{\beta}_{k j} \frac{I_{j}}{I_{j}^{*}}-\sum_{j=1}^{n} \bar{\beta}_{k j} \frac{I_{k}}{I_{k}^{*}}\right)=0, \\
\sum_{k=1}^{n} \bar{c}_{k}\left(\sum_{j=1}^{n} \bar{\beta}_{k j} \ln \frac{I_{j}}{I_{j}^{*}}-\sum_{j=1}^{n} \bar{\beta}_{k j} \ln \frac{I_{k}}{I_{k}^{*}}\right)=0 .
\end{gathered}
$$

Besides, note that $x-1-\ln x \geq 0$ for $x>0$; then

$$
\frac{S_{k}^{*}}{S_{k}} \geq 1+\ln \frac{S_{k}^{*}}{S_{k}}, \quad \frac{S_{k} I_{j} I_{k}^{*}}{I_{k} S_{k}^{*} I_{j}^{*}} \geq 1+\ln \frac{S_{k} I_{j} I_{k}^{*}}{I_{k} S_{k}^{*} I_{j}^{*}} .
$$

According to (41) and (42), we get

$$
\begin{aligned}
& \sum_{k=1}^{n} \bar{c}_{k} \sum_{j=1}^{n} \bar{\beta}_{k j}\left(2-\frac{S_{k}^{*}}{S_{k}}-\frac{S_{k} I_{j} I_{k}^{*}}{I_{k} S_{k}^{*} I_{j}^{*}}\right) \\
& \leq \sum_{k=1}^{n} \bar{c}_{k} \sum_{j=1}^{n} \bar{\beta}_{k j}\left[2-\left(1+\ln \frac{S_{k}^{*}}{S_{k}}\right)-\left(1+\ln \frac{S_{k} I_{j} I_{k}^{*}}{I_{k} S_{k}^{*} I_{j}^{*}}\right)\right] \\
& =\sum_{k=1}^{n} \bar{c}_{k} \sum_{j=1}^{n} \bar{\beta}_{k j}\left(\ln \frac{I_{k}}{I_{k}^{*}}-\ln \frac{I_{j}}{I_{j}^{*}}\right)=0,
\end{aligned}
$$




$$
\begin{aligned}
\sum_{k=1}^{n} \bar{c}_{k} \sum_{j=1}^{n} \bar{\beta}_{k j} \frac{S_{k} I_{j} I_{k}^{*}}{I_{k} S_{k}^{*} I_{j}^{*}} \geq & \sum_{k=1}^{n} \bar{c}_{k} \sum_{j=1}^{n} \bar{\beta}_{k j}\left(1+\ln \frac{S_{k} I_{j} I_{k}^{*}}{I_{k} S_{k}^{*} I_{j}^{*}}\right) \\
= & \sum_{k=1}^{n} \bar{c}_{k} \sum_{j=1}^{n} \bar{\beta}_{k j}\left(1-\ln \frac{S_{k}^{*}}{S_{k}}\right) \\
& +\sum_{k=1}^{n} \bar{c}_{k} \sum_{j=1}^{n} \bar{\beta}_{k j}\left(\ln \frac{I_{j}}{I_{j}^{*}}-\ln \frac{I_{k}}{I_{k}^{*}}\right) \\
\geq & \sum_{k=1}^{n} \bar{c}_{k} \sum_{j=1}^{n} \bar{\beta}_{k j}\left(2-\frac{S_{k}^{*}}{S_{k}}\right) .
\end{aligned}
$$

Substituting (41) and (43) into (38), we get

$$
\begin{aligned}
L V_{1} & \leq \sum_{k=1}^{n} \bar{c}_{k}\left[-d S_{k}^{*}\left(\frac{S_{k}^{*}}{S_{k}}+\frac{S_{k}}{S_{k}^{*}}-2\right)+\frac{1}{2}\left(S_{k}^{*} \sigma_{k 1}^{2}+I_{k}^{*} \sigma_{k 2}^{2}\right)\right] \\
& =-\sum_{k=1}^{n} \bar{c}_{k} d \frac{\left(S_{k}-S_{k}^{*}\right)^{2}}{S_{k}}+\sum_{k=1}^{n} \frac{\bar{c}_{k}}{2}\left(S_{k}^{*} \sigma_{k 1}^{2}+I_{k}^{*} \sigma_{k 2}^{2}\right) .
\end{aligned}
$$

Substituting (44) into (39), we get

$$
\begin{aligned}
L V_{2} \leq \sum_{k=1}^{n} \bar{c}_{k}\left[\sum_{j=1}^{n} \beta_{k j} S_{k} I_{j}-\sum_{j=1}^{n} \bar{\beta}_{k j} \frac{I_{k}}{I_{k}^{*}}-\sum_{j=1}^{n} \bar{\beta}_{k j}\left(2-\frac{S_{k}^{*}}{S_{k}}\right)\right. & \\
& \left.+\sum_{j=1}^{n} \bar{\beta}_{k j}+\frac{I_{k}^{*} \sigma_{k 2}^{2}}{2}\right] \\
=\sum_{k=1}^{n} \bar{c}_{k}\left[\sum_{j=1}^{n} \beta_{k j}\left(S_{k}-S_{k}^{*}\right)\left(I_{j}-I_{j}^{*}\right)\right. & -\sum_{j=1}^{n} \bar{\beta}_{k j}\left(\frac{I_{k}}{I_{k}^{*}}-\frac{I_{j}}{I_{j}^{*}}\right) \\
& \left.-\sum_{j=1}^{n} \bar{\beta}_{k j}\left(2-\frac{S_{k}^{*}}{S_{k}}-\frac{S_{k}}{S_{k}^{*}}\right)+\frac{I_{k}^{*} \sigma_{k 2}^{2}}{2}\right] \\
=\sum_{k=1}^{n} \bar{c}_{k}\left[\sum_{j=1}^{n} \beta_{k j}\left(S_{k}-S_{k}^{*}\right)\left(I_{j}-I_{j}^{*}\right)\right. & \left.+\sum_{j=1}^{n} \beta_{k j} I_{j}^{*} \frac{\left(S_{k}-S_{k}^{*}\right)^{2}}{S_{k}}+\frac{I_{k}^{*} \sigma_{k 2}^{2}}{2}\right] .
\end{aligned}
$$

Therefore,

$$
\begin{aligned}
L V & =a L V_{1}+L V_{2}+L V_{3}+L V_{4} \\
& \leq-\sum_{k=1}^{n}\left[p_{k} d-m_{k} \frac{(d+\epsilon)^{2}+d^{2}}{2(d+\epsilon)}\right]\left(S_{k}-S_{k}^{*}\right)^{2}
\end{aligned}
$$

$$
\begin{aligned}
& -\frac{1}{2} \sum_{k=1}^{n} m_{k}(d+\epsilon)\left(I_{k}-I_{k}^{*}\right)^{2} \\
& -\sum_{k=1}^{n} \bar{c}_{k}\left(a d-\sum_{j=1}^{n} \beta_{k j} I_{j}^{*}\right) \frac{\left(S_{k}-S_{k}^{*}\right)^{2}}{S_{k}} \\
& +\sum_{k=1}^{n} \sum_{j=1}^{n} \beta_{k j}\left(\bar{c}_{k}-p_{k} S_{k}^{*}\right)\left(S_{k}-S_{k}^{*}\right)\left(I_{j}-I_{j}^{*}\right) \\
& -\sum_{k=1}^{n} \sum_{j=1}^{n} p_{k} \beta_{k j} I_{j}\left(S_{k}-S_{k}^{*}\right)^{2} \\
& +\sum_{k=1}^{n} \frac{a \bar{c}_{k} S_{k}^{*}+\left(m_{k}+p_{k}\right) S_{k}^{2}}{2} \sigma_{k 1}^{2} \\
& +\sum_{k=1}^{n} \frac{(a+1) \bar{c}_{k} I_{k}^{*}+m_{k} I_{k}^{2}}{2} \sigma_{k 2}^{2} \\
& \leq-\sum_{k=1}^{n}\left[p_{k} d-m_{k} \frac{(d+\epsilon)^{2}+d^{2}}{2(d+\epsilon)}\right]\left(S_{k}-S_{k}^{*}\right)^{2} \\
& -\frac{1}{2} \sum_{k=1}^{n} m_{k}(d+\epsilon)\left(I_{k}-I_{k}^{*}\right)^{2} \\
& -\sum_{k=1}^{n} \bar{c}_{k}\left(a d-\sum_{j=1}^{n} \beta_{k j} I_{j}^{*}\right) \frac{\left(S_{k}-S_{k}^{*}\right)^{2}}{S_{k}} \\
& +\sum_{k=1}^{n} \sum_{j=1}^{n} \beta_{k j}\left(\bar{c}_{k}-p_{k} S_{k}^{*}\right)\left(S_{k}-S_{k}^{*}\right)\left(I_{j}-I_{j}^{*}\right) \\
& +\sum_{k=1}^{n} \frac{a \bar{c}_{k} S_{k}^{*}+\left(m_{k}+p_{k}\right) S_{k}^{2}}{2} \sigma_{k 1}^{2} \\
& +\sum_{k=1}^{n} \frac{(a+1) \bar{c}_{k} I_{k}^{*}+m_{k} I_{k}^{2}}{2} \sigma_{k 2}^{2} .
\end{aligned}
$$

Choose $a=\max \left\{\left(\sum_{j=1}^{n} \beta_{k j} I_{j}^{*}\right) / d, k=1,2, \ldots, n\right\}, m_{k}=(d+$ e) $p_{k} d /\left((d+\epsilon)^{2}+d^{2}\right), p_{k}=\bar{c}_{k} / S_{k}^{*}, k=1,2, \ldots, n$; then

$$
\begin{gathered}
L V \leq-\sum_{k=1}^{n} \frac{p_{k} d}{2}\left(S_{k}-S_{k}^{*}\right)^{2}-\frac{1}{2} \sum_{k=1}^{n} m_{k}(d+\epsilon)\left(I_{k}-I_{k}^{*}\right)^{2} \\
+\frac{1}{2} \sum_{k=1}^{n}\left[\left(\frac{a \bar{c}_{k} b}{d}+\frac{\left(m_{k}+p_{k}\right) b^{2}}{d^{2}}\right) \sigma_{k 1}^{2}\right. \\
\left.+\left(\frac{(a+1) \bar{c}_{k} b}{d}+\frac{m_{k} b^{2}}{d^{2}}\right) \sigma_{k 2}^{2}\right]
\end{gathered}
$$

$:=F(t)$ 
Therefore,

$d V \leq F(t) d t$

$$
\begin{aligned}
+\sum_{k=1}^{n}[ & {\left[a \bar{c}_{k} \sigma_{k 1}\left(S_{k}-S_{k}^{*}\right)+\left(m_{k}+p_{k}\right) \sigma_{k 1}\left(S_{k}-S_{k}^{*}\right) S_{k}\right] } \\
& \times d B_{k 1}(t) \\
& +\left[\bar{c}_{k}(a+1) \sigma_{k 2}\left(I_{k}-I_{k}^{*}\right)+m_{k} \sigma_{k 2}\left(I_{k}-I_{k}^{*}\right) I_{k}\right] \\
& \left.\times d B_{k 2}(t)\right] .
\end{aligned}
$$

Integrating both sides of (49) from 0 to $t$ yields

$$
\begin{aligned}
& V(t)-V(0) \\
& \leq \int_{0}^{t} F(s) d s \\
& \quad+\int_{0}^{t} \sum_{k=1}^{n}\left[a \bar{c}_{k} \sigma_{k 1}\left(S_{k}-S_{k}^{*}\right)+\left(m_{k}+p_{k}\right) \sigma_{k 1}\left(S_{k}-S_{k}^{*}\right) S_{k}\right] \\
& \quad \times d B_{k 1}(s) \\
& \quad+\int_{0}^{t} \sum_{k=1}^{n}\left[\bar{c}_{k}(a+1) \sigma_{k 2}\left(I_{k}-I_{k}^{*}\right)+m_{k} \sigma_{k 2}\left(I_{k}-I_{k}^{*}\right) I_{k}\right] \\
& \quad \times d B_{k 2}(s) .
\end{aligned}
$$

Let $M_{1}(t):=\int_{0}^{t} \sum_{k=1}^{n}\left[a \bar{c}_{k} \sigma_{k 1}\left(S_{k}-S_{k}^{*}\right)+\left(m_{k}+p_{k}\right) \sigma_{k 1}\left(S_{k}-\right.\right.$ $\left.\left.S_{k}^{*}\right) S_{k}\right] d B_{k 1}(s), M_{2}(t):=\int_{0}^{t} \sum_{k=1}^{n}\left[\bar{c}_{k}(a+1) \sigma_{k 2}\left(I_{k}-I_{k}^{*}\right)+\right.$ $\left.m_{k} \sigma_{k 2}\left(I_{k}-I_{k}^{*}\right) I_{k}\right] d B_{k 2}(s)$, which are local continuous martingale, and $M_{1}(0)=M_{2}(0)=0$. Moreover

$$
\begin{aligned}
& \limsup _{t \rightarrow \infty} \frac{\left\langle M_{1}, M_{1}\right\rangle_{t}}{t} \leq 8 \sum_{k=1}^{n} \sigma_{k 1}^{2}\left[a^{2} \bar{c}_{k}^{2}+\left(m_{k}+p_{k}\right)^{2} \frac{b_{k}^{2}}{d^{2}}\right] \frac{b_{k}^{2}}{d^{2}} \\
&<\infty, \\
& \limsup _{t \rightarrow \infty} \frac{\left\langle M_{2}, M_{2}\right\rangle_{t}}{t} \leq 8 \sum_{k=1}^{n} \sigma_{k 2}^{2}\left[\bar{c}_{k}^{2}(a+1)^{2}+m_{k}^{2} \frac{b_{k}^{2}}{d^{2}}\right] \frac{b_{k}^{2}}{d^{2}}<\infty .
\end{aligned}
$$

By Lemma A.4 (see [20]), we obtain

$$
\lim _{t \rightarrow \infty} \frac{M_{1}(t)}{t}=0, \quad \lim _{t \rightarrow \infty} \frac{M_{2}(t)}{t}=0 \quad \text { a.s., }
$$

which together with (50) implies

$$
\liminf _{t \rightarrow \infty} \frac{\int_{0}^{t} F(s) d s}{t} \geq 0 \text { a.s. }
$$

Consequently,

$$
\begin{gathered}
\limsup _{t \rightarrow \infty} \frac{1}{t} \sum_{k=1}^{n} \int_{0}^{t}\left[p_{k} d\left(S_{k}-S_{k}^{*}\right)^{2}+m_{k}(d+\epsilon)\left(I_{k}-I_{k}^{*}\right)^{2}\right] d s \\
\leq \sum_{k=1}^{n}\left[\left(\frac{a \bar{c}_{k} b_{k}}{d}+\frac{\left(m_{k}+p_{k}\right) b_{k}^{2}}{d^{2}}\right) \sigma_{k 1}^{2}\right. \\
\left.+\left(\frac{(a+1) \bar{c}_{k} b_{k}}{d}+\frac{m_{k} b_{k}^{2}}{d^{2}}\right) \sigma_{k 2}^{2}\right], \quad \text { a.s. }
\end{gathered}
$$

Thus Theorem 5 is proved.

Remark 6. Theorem 5 shows that the solution of system (5) fluctuates around the certain level which is relevant to $E^{*}$ of system (3) and $\sigma_{k 1}^{2}, \sigma_{k 2}^{2}, k=1,2, \ldots, n$. The distance between the solution $X(t)=\left(S_{1}(t), I_{1}(t), \ldots, S_{n}(t), I_{n}(t)\right)$ and $E^{*}$ of system (3) has the following form:

$$
\limsup _{t \rightarrow \infty} \frac{1}{t} \int_{0}^{t}\left\|X(s)-E^{*}\right\|^{2} d s \leq C\|\sigma\|^{2}
$$

where $C$ is a positive constant and $\|\sigma\|^{2}=\sum_{k=1}^{n}\left(\sigma_{k 1}^{2}+\sigma_{k 2}^{2}\right)$. Although the solution of system (5) does not have stability as the deterministic system, we can draw a conclusion that system (5) is persistent on the basis of the result of Theorem 5 , which also accounts for the fact that the disease is prevalent.

\section{Simulations and Conclusions}

5.1. Numerical Simulations. In order to confirm the results above, we numerically simulate the solution of system (5) with $n=50, b_{k}=0.25, d=0.3, \epsilon=0.01$, and initial value $S_{k}(0)=0.5, I_{k}(0)=0.1, k=1,2, \ldots, 50$. Using Milstein's Higer Order Method [31], we get the discretization equation:

$$
\begin{aligned}
S_{k, i+1}= & S_{k, i}+\Delta t\left(b_{k}-\sum_{j=1}^{2} \beta_{k j} S_{k, i} I_{j, i}-d S_{k, i}\right) \\
& +\sigma_{k 1} S_{k, i} \sqrt{\Delta t} \xi_{k 1, i}+\frac{\sigma_{k 1}^{2}}{2} S_{k, i} \Delta t\left(\xi_{k 1, i}^{2}-1\right), \\
I_{k, i+1}= & I_{k, i}+\Delta t\left(\sum_{j=1}^{2} \beta_{k j} S_{k, i} I_{j, i}-(d+\epsilon) I_{k, i}\right) \\
& +\sigma_{k 2} I_{k, i} \sqrt{\Delta t} \xi_{k 2, i}+\frac{\sigma_{k 2}^{2}}{2} I_{k, i} \Delta t\left(\xi_{k 2, i}^{2}-1\right),
\end{aligned}
$$

where $k=1,2, \ldots, n$ and $\xi_{k 1, i}, \xi_{k 2, i}, i=1,2, \ldots, N$, are the independent Gaussian random variables $N(0,1)$.

From Theorem 3 and Remark 4, it is shown that the expectations of $S_{k}(t), I_{k}(t), k=1,2, \ldots, n$, are converging under some conditions, and the solution of system (5) will oscillate around the disease-free equilibrium of system (3). In Figure 1, we choose parameters $\lambda=0.025, \sigma_{k 1}=0.005$, and $\sigma_{k 2}=0.03$, such that $R_{0} \leq 1$, and in Figures 1(a) and 1(b) we choose $P(k)=m^{k} \exp (-m) / k$ !, and in Figures $1(\mathrm{c})$ and $1(\mathrm{~d})$ 
we choose $P(k)=2 m^{2} k^{-3}$. From Figure 1, we can see that the disease-free equilibrium $E_{0}$ of system (3) (imaginary lines) is globally asymptotically stable and the curves of system (5) (real lines) always fluctuate around the curves of system (3) (imaginary lines). From Figure 2, we can see that, due to the difference of the degree distribution, the critical value of spread is different.

In Figure 3, parameters $\lambda=0.08, \sigma_{k 1}=0.01$, and $\sigma_{k 2}=0.01$ and others are the same as the previous. From Figure 3, we can see that the position of the equilibrium state is different due to the difference of the degree distribution. From Figures 1(d), 2(b), 4(b), and 4(d), we found that the solution of stochastic system converging to the disease-free equilibrium is faster than that of the deterministic system with the increase of noise intensity.

5.2. Conclusions. The numerical simulations illustrate the mathematical theorems well. Due to the existence of the noise, the solution of the stochastic system goes around the solution of the deterministic system. With intensities decreasing, the turbulence intensity is weaker. From numerical simulations, we have a new discovery. When $R_{0} \leq 1$, with the increase of noise intensity, the solution of stochastic system converging to the disease-free equilibrium is faster than the deterministic system. This is because of the fact that, when $R_{0} \leq 1$, the disease will die out after some time. However in the real world many stochastic factors contributed to the extinction of the disease.

\section{Conflict of Interests}

The authors declare that there is no conflict of interests regarding the publication of this paper.

\section{Acknowledgments}

The authors would like to thank the anonymous referees for their useful feedback, helpful comments, and suggestions which have improved the paper. This project is supported by the National Sciences Foundation of China (10901145, 11331009, 11301491), the Top Young Academic Leaders of Higher Learning Institutions of Shanxi, and the Sciences Foundation of Shanxi Province (2012011002-1).

\section{References}

[1] N. T. Bailey, The Mathematical Theory of Infectious Disease, Hafner Press, New York, NY, USA, 2nd edition, 1975.

[2] R. M. Anderson and R. M. May, Infectious Diseases of Humans, Oxford University Press, Oxford, UK, 1992.

[3] O. Diekmann, J. A. P. Heesterbeek, and J. A. J. Metz, “On the definition and the computation of the basic reproduction ratio $R_{0}$ in models for infectious diseases in heterogeneous populations," Journal of Mathematical Biology, vol. 28, no. 4, pp. 365-382, 1990.

[4] O. Diekmann and J. A. P. Heesterbeek, Mathematical Epidemiology of Infectious Diseases, John Wiley \& Sons, Chichester, UK, 2000, Model building, analysis and interpretation.
[5] H. W. Hethcote, "The mathematics of infectious diseases," SIAM Review, vol. 42, no. 4, pp. 599-653, 2000.

[6] J. Wang, M. Liu, and Y. Li, "Analysis of epidemic models with demographics in metapopulation networks," Physica A, vol. 392, no. 7, pp. 1621-1630, 2013.

[7] S. Eubank, H. Guclu, V. S. A. Kumar et al., "Modelling disease outbreaks in realistic urban social networks," Nature, vol. 429, no. 6988, pp. 180-184, 2004.

[8] M. Barthélemy, A. Barrat, R. Pastor-Satorras, and A. Vespignani, "Dynamical patterns of epidemic outbreaks in complex heterogeneous networks," Journal of Theoretical Biology, vol. 235, no. 2, pp. 275-288, 2005.

[9] L. Wang and G.-Z. Dai, "Global stability of virus spreading in complex heterogeneous networks," SIAM Journal on Applied Mathematics, vol. 68, no. 5, pp. 1495-1502, 2008.

[10] L. Mao-Xing and R. Jiong, "Modelling the spread of sexually transmitted diseases on scale-free networks," Chinese Physics B, vol. 18, no. 6, pp. 2115-2120, 2009.

[11] R. Pastor-Satorras and A. Vespignani, "Epidemic spreading in scale-free networks," Physical Review Letters, vol. 86, no. 14, pp. 3200-3203, 2001.

[12] R. Pastor-Satorras and A. Vespignani, "Epidemic dynamics in finite size scale-free networks," Physical Review E, vol. 65, no. 3, Article ID 035108, pp. 035108/1-035108/4, 2002.

[13] J.-p. Zhang and Z. Jin, "The analysis of an epidemic model on networks," Applied Mathematics and Computation, vol. 217, no. 17, pp. 7053-7064, 2011.

[14] J. Z. Liu, Y. F. Tang, and Z. R. Yang, "The spread of disease with birth and death on networks," Journal of Statistical Mechanics, vol. 2004, Article ID P08008, 2004.

[15] X. Fu, M. Small, D. M. Walker, and H. Zhang, "Epidemic dynamics on scale-free networks with piecewise linear infectivity and immunization," Physical Review E, vol. 77, no. 3, pp. 036113/1-036113/8, 2008.

[16] T. Zhou, J.-G. Liu, W.-J. Bai, G. Chen, and B.-H. Wang, "Behaviors of susceptible-infected epidemics on scale-free networks with identical infectivity," Physical Review E, vol. 74, no. 5, pp. 056109/1-056109/6, 2006.

[17] M.-X. Liu and J. Ruan, "A stochastic epidemic model on homogeneous networks," Chinese Physics B, vol. 18, no. 12, pp. 5111-5116, 2009.

[18] C. Q. Xu, S. L. Yuan, and T. H. Zhang, "Asymptotic behavior of a chemostat model with stochastic perturbation on the dilution rate," Abstract and Applied Analysis, vol. 2013, Article ID 423154, 11 pages, 2013.

[19] D. Jiang, J. Yu, C. Ji, and N. Shi, "Asymptotic behavior of global positive solution to a stochastic SIR model," Mathematical and Computer Modelling, vol. 54, no. 1-2, pp. 221-232, 2011.

[20] C. Ji, D. Jiang, and N. Shi, "Multigroup SIR epidemic model with stochastic perturbation," Physica A, vol. 390, no. 10, pp. 17471762, 2011.

[21] C. Yuan, D. Jiang, D. O’Regan, and R. P. Agarwal, "Stochastically asymptotically stability of the multi-group SEIR and SIR models with random perturbation," Communications in Nonlinear Science and Numerical Simulation, vol. 17, no. 6, pp. 2501-2516, 2012.

[22] E. Tornatore, S. M. Buccellato, and P. Vetro, "Stability of a stochastic SIR system," Physica A, vol. 354, no. 1-4, pp. 111-126, 2005.

[23] P. J. Witbooi, "Stability of an SEIR epidemic model with independent stochastic perturbations," Physica A, vol. 392, no. 20, pp. 4928-4936, 2013. 
[24] Y. Zhao, D. Jiang, and D. O’Regan, “The extinction and persistence of the stochastic SIS epidemic model with vaccination," Physica A, vol. 392, no. 20, pp. 4916-4927, 2013.

[25] G. Hu, M. Liu, and K. Wang, "The asymptotic behaviours of an epidemic model with two correlated stochastic perturbations," Applied Mathematics and Computation, vol. 218, no. 21, pp. 10520-10532, 2012.

[26] A. Lahrouz and L. Omari, "Extinction and stationary distribution of a stochastic SIRS epidemic model with non-linear incidence," Statistics \& Probability Letters, vol. 83, no. 4, pp. 960968, 2013.

[27] L. Arnold, Stochastic Differential Equations: Theory and Applications, Wiley Press, New York, NY, USA, 1974.

[28] X. Mao, Stochastic Differential Equations and Applications, Horwood Press, Chichester, UK, 1997.

[29] N. Dalal, D. Greenhalgh, and X. Mao, "A stochastic model of AIDS and condom use," Journal of Mathematical Analysis and Applications, vol. 325, no. 1, pp. 36-53, 2007.

[30] X. Mao, G. Marion, and E. Renshaw, "Environmental brownian noise suppresses explosions in population dynamics," Stochastic Processes and their Applications, vol. 97, no. 1, pp. 95-110, 2002.

[31] D. J. Higham, "An algorithmic introduction to numerical simulation of stochastic differential equations," SIAM Review, vol. 43 , no. 3 , pp. 525-546, 2001. 


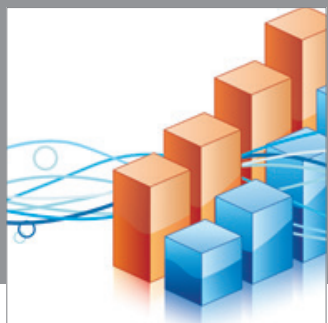

Advances in

Operations Research

mansans

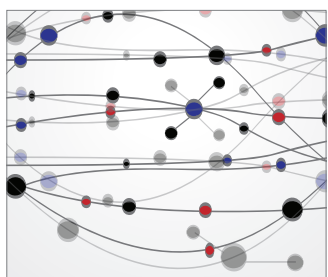

The Scientific World Journal
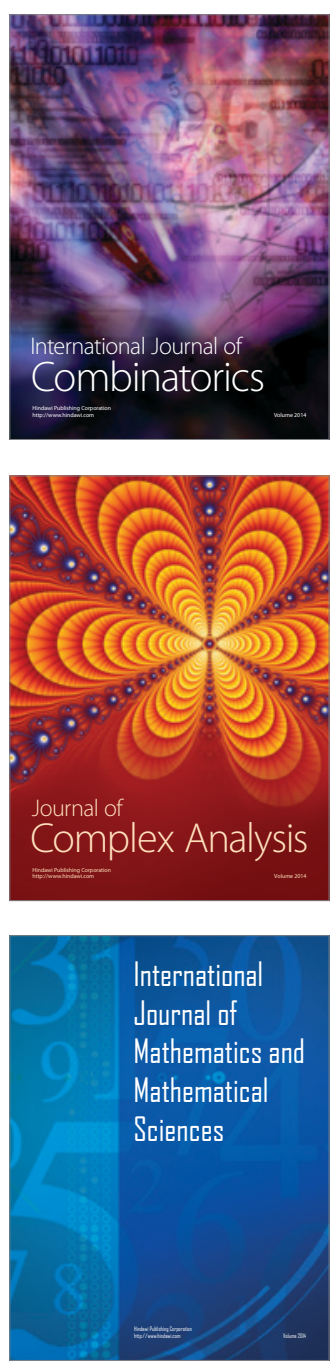
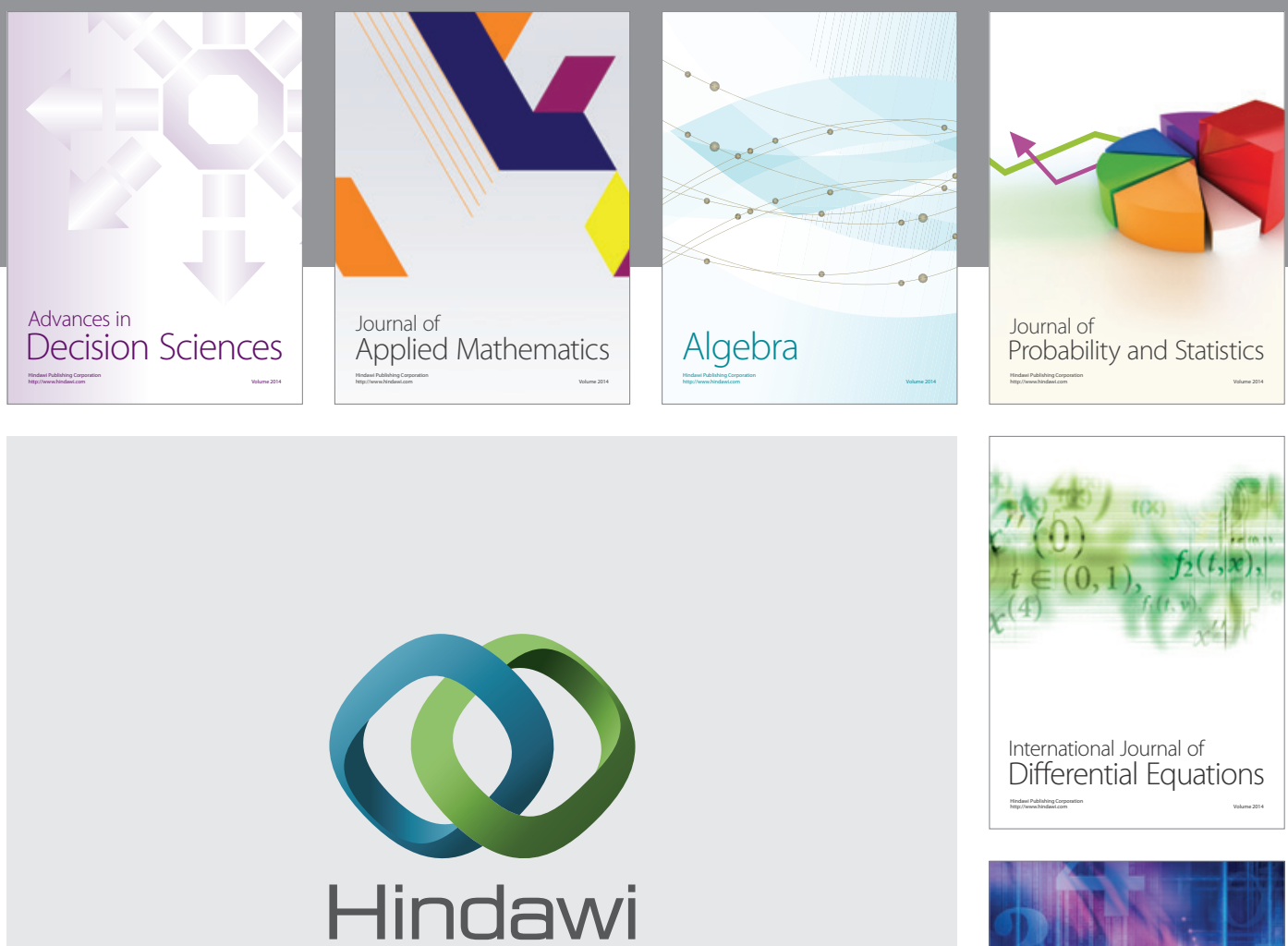

Submit your manuscripts at http://www.hindawi.com
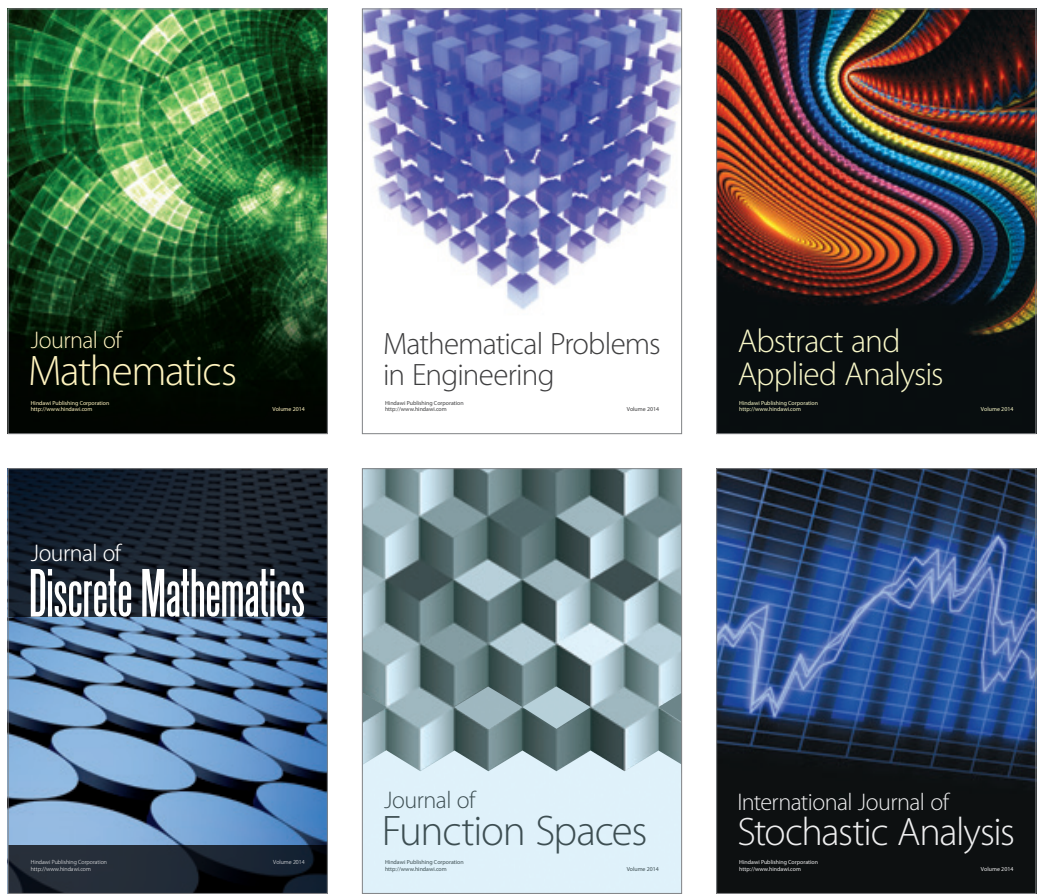

Journal of

Function Spaces

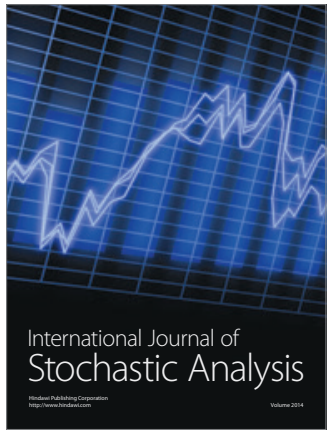

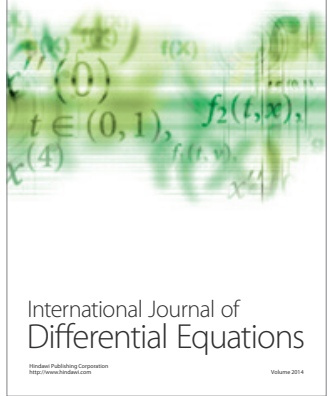
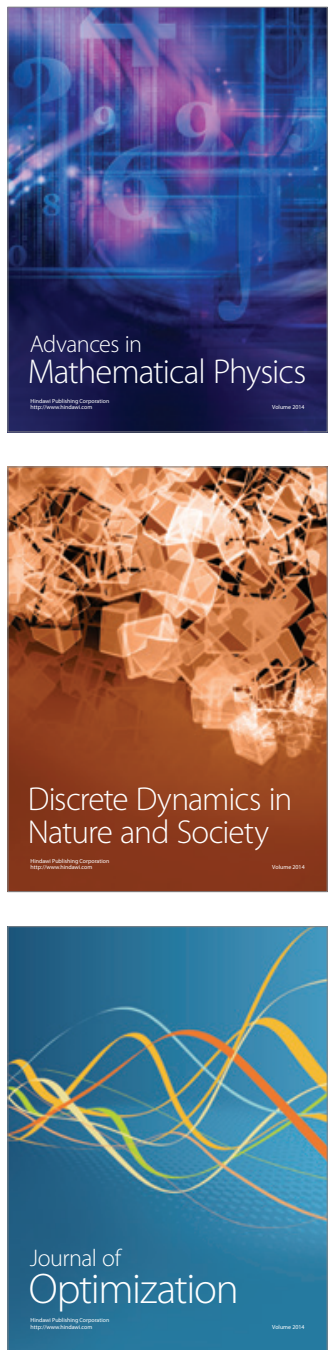